\title{
OPEN Olfactomedin 4 mediation of prostate stem/progenitor-like cell proliferation and differentiation via MYC
}

\author{
Hongzhen Li ${ }^{1}$, Vijender Chaitankar ${ }^{2}$, Jianqiong Zhu' ${ }^{1}$, Kyung Chin ${ }^{1}$, Wenli Liu ${ }^{1}$, \\ Mehdi Pirooznia $^{2}$ \& Griffin P. Rodgers ${ }^{1 凶}$
}

Olfactomedin 4 (OLFM4) is expressed in normal prostate epithelial cells and immortalized normal human prostate epithelial cells (RWPE1), but the identity of OLFM4-expressing cells within these populations and OLFM4's physiological functions in these cells have not been elucidated. Using singlecell RNA sequencing analysis, we found here that OLFM4 was expressed in multiple stem/progenitorlike cell populations in both the normal prostate epithelium and RWPE1 cells and was frequently co-expressed with KRT13 and LY6D in RWPE1 cells. Functionally, OLFM4-knockout RWPE1 cells exhibited enhanced proliferation of the stem/progenitor-like cell population, shifts stem/progenitorlike cell division to favor symmetric division and differentiated into higher levels PSA expression cells in organoid assays compared with OLFM4-wild RWPE1 cells. Bulk-cell RNA sequencing analysis pinpointed that $C M Y C$ expression were enhanced in the OLFM4-knockout RWPE1 cells compared with OLFM4-wild cells. Molecular and signaling pathway studies revealed an increase in the WNT/APC/ MYC signaling pathway gene signature, as well as that of $M Y C$ target genes that regulate multiple biological processes, in OLFM4-knockout RWPE1 cells. These findings indicated that OLFM4 is co-expressed with multiple stem/progenitor cell marker genes in prostate epithelial cells and acts as a novel mediator in prostate stem/progenitor cell proliferation and differentiation.

Prostate stem/progenitor cells exist within prostatic pseudostratified epithelium and exhibit the capacities of self-renewal and multi-lineage differentiation that underly prostate organogenesis and homeostasis. The stem cells self-renew via one stem cell giving rise to two stem cells (symmetric cell division) and differentiate via one stem cell giving rise to one stem-cell copy and one progenitor cell (asymmetric cell division), and the one progenitor cell subsequently giving rise to two differentiated cells (committed cell division) ${ }^{1-3}$. Studies of prostate stem/progenitor cells have been performed using cell-surface markers, such as CD133, CD44, CD49F, CD24, and CD26, that are found on enriched putative stem/progenitor cells in human and mouse normal or tumor prostate tissues $^{4-6}$. Recently, cytokeratin (CK) 13 (KRT13) has been identified as a marker for normal human prostate epithelial stem/progenitor cells, as well as bone marrow metastatic cancer cells ${ }^{7-9}$. The cytokeratins and other specific molecular markers have been used as epithelial cell markers for identifying cell types in the prostate epithelium. For example, CK5, CK8, CK18, and CK 19 are intermediate cell markers, CK5, CK14, and p63 are basal cell markers, and CK8, CK18, and androgen receptor (AR) are luminal cell markers; synaptophysin serves as a rare neuroendocrine cell marker in the developing and adult prostate epithelium ${ }^{10-13}$.

Experimental studies of properties of stem/progenitor cells have been performed using prostate regeneration, tissue recombination, and genetic marker tracing in rodent models ${ }^{14-16}$. Studies of human prostate epithelial stem/progenitor cells have been performed using primary two-dimensional (2D) cell culture, Matrigel three-dimensional (3D) cell culture, and combination xenograft ex vivo models ${ }^{12,17,18}$. Recently, single-cell RNA sequencing technology has been performed for identifying putative stem/progenitor cells in normal adult prostate epithelium and in primary-culture human benign prostate epithelial cells ${ }^{8,9}$. We have also previously identified putative prostate stem/progenitor cells in immortalized human normal and benign prostate epithelial cell

\footnotetext{
${ }^{1}$ Molecular and Clinical Hematology Branch, National Heart, Lung, and Blood Institute, National Institutes of Health, Bldg. 10, Room 9N119, 9000 Rockville Pike, Bethesda, MD 20892, USA. ${ }^{2}$ Bioinformatics and Systems Biology Core, National Heart, Lung, and Blood Institute, National Institutes of Health, Bethesda, MD 20892,

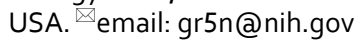


populations ${ }^{19,20}$. The RWPE1 immortalized human normal prostate epithelial cell line ${ }^{21}$ that we used in those studies has been widely used as a benign prostate epithelial cell line for molecular, cellular, and biological studies.

The WNT/APC/MYC signaling pathways play an important role in prostate development and prostate-cancer progression by mediating prostate stem/progenitor cell functions ${ }^{22,23}$. MYC protein serves as a master regulator of cell proliferation, metabolism, ribosome biogenesis, protein synthesis, and mitochondrial function ${ }^{24}$. MYC protein targets most cell-cycle regulator genes, including cyclins, cyclin-dependent kinases, and cell-cycle inhibitors, drives quiescent cells to enter the cell cycle, and promotes cell proliferation ${ }^{24,25}$. The MYC oncogene is somatically amplified in a subset of advanced prostate cancer ${ }^{26}$. Overexpression of MYC mRNA has been found in both prostate intraepithelial neoplasia and $\operatorname{carcinomas}^{27,28}$, and overexpression of MYC protein has been reported as an early alteration in human prostate carcinogenesis ${ }^{29}$. The $M y c$-driven murine prostate-cancer model mimics the progression of human prostate cancer, in which MYC is overexpressed ove,31. $^{30}$.

The olfactomedin 4 (OLFM4) gene was first cloned from human myeloid progenitor cells and is normally expressed in prostate, bone marrow, small intestine, and pancreas ${ }^{32}$. The OLFM4 gene plays an important role in innate immunity, inflammation, and cancers ${ }^{33}$. We have reported previously that OLFM4 gene expression was reduced or lost during the progression of prostate cancer due to frequent genetic deletion ${ }^{34}$ and hypermethylation of the OLFM4 gene promoter region ${ }^{35}$. The expression of OLFM4 mRNA has been detected in normal prostate tissues, primary-cultured normal prostate epithelial cells, and RWPE1 immortalized normal human prostate epithelial cells ${ }^{34-36}$. However, OLFM4-expressing cell subtypes and OLFM4's physiological functions in those cells remain elusive.

In this study, we sought to identify and characterize the OLFM4-expressing cells found within prostate epithelial cells. We identified OLFM4-expressing cells as belonging to multiple stem/progenitor cell populations in both the normal prostate epithelium and in RWPE1 cells. We demonstrated that knockout of the OLFM4 gene in RWPE1 cells enhanced the proliferation of stem/progenitor-like cell populations, shifts stem/progenitor-like cell division to favor symmetric division, and differentiated into higher levels PSA expression cells in organoid assays when compared with OLFM4-wild RWPE1 cells. Furthermore, gene set enrichment analysis of RNA-sequencing results for RWPE1 stem/progenitor-like cells revealed that OLFM4 knockout enriched gene signatures related to stem cells, which were subsequently identified to be related to the WNT/APC/MYC signaling pathway. Taken together, our results suggest that OLFM4-expressing prostate epithelial cells represent multiple stem/progenitor cell populations and that OLFM4 mediates those cells' proliferation and differentiation through the WNT/APC/ MYC signaling pathway.

\section{Results}

OLFM4 is highly co-expressed with stem/progenitor cell markers in normal human adult prostate epithelium. To identify OLFM4-expressing cells in the normal human prostate, we downloaded a dataset from single-cell RNA sequencing for normal human prostate (GSE117403, which is a part of super series GSE $120716^{8}$ ) and analyzed cells for gene-expression signatures using Uniform Manifold Approximation and Projection (UMAP) software. Analysis of differentially expressed genes confirmed 4 epithelial clusters and 4 stromal clusters (leukocyte, endothelium, fibroblast, and smooth muscle) within 19 clusters (Fig. 1a, left panel) ${ }^{8}$. Higher populations of OLFM4-expressing cells were confirmed in clusters 7 and 12 and lower populations were identified in four other epithelial clusters (Fig. 1a, right panel and Supplementary Table S1). OLFM4 was highly co-expressed with SCGB3A1, LCN2, PIGR, PSCA (prostate stem cell antigen), and CD24, which are all prostate stem-cell markers, in cluster 7, and with KRT13, APOBEC3A, LYPD3, and KRT19, which are all prostate stem/ progenitor cell markers, in cluster 12 (Fig. 1a, right panel, Supplementary Fig. S1, and Table S1). Cluster 7 has previously been reported to belong to Club cells, which express SCGB1A1, and cluster 12 has previously been reported to belong to Hillock epithelial cells, which express higher levels of KRT13 markers in the human normal prostate epithelia ${ }^{8}$ and in the mouse lung epithelia ${ }^{37}$.

We further classified OLFM4-expressing prostate epithelial cells by using markers for stem/progenitor cells (KRT13 [CK13] for cluster 12 cells, SCGB1A1 for cluster 7 cells and CD44+ cells); basal cells (CK5, CK14); and luminal cells (CK8). Triple-color immunofluorescent staining with specific antibodies demonstrated the expression of OLFM4 in the CK13+, CD44+, and CK5+ cells, where multiple layers of epithelia are found in the prostatic urethra tube (Fig. 1b). We observed about 6\% (12/187) cells are expressing SCGB1A1 among OLFM4 positive cells in the prostatic urethra tube and peri-urethra tube epithelia (Fig. 1c). OLFM4+/CK8+ cells were found within multiple layers of epithelia in the prostatic urethra and peri-urethra tube and a few in the distal regions of the prostatic gland (Supplementary Fig. S2). Further, OLFM4+/CK8+/FOXA1+ cells were observed in the distal regions of prostatic gland (Supplementary Fig. S2) and prostatic acini (Supplementary Fig. S2). Doublecolor immunofluorescent staining showed that OLFM4+/CD44+ cells are distributed within CD44+ epithelial cells in the prostate acini (Supplementary Fig. S2). OLFM4+/CK14- and OLFM4+/CK8 - cells are present within epithelial cells in the prostate acini (Supplementary Fig. S2). Taken together, these results suggest that OLFM4expressing cells are CK13+/CD44+, SCGB1A1+ stem/progenitor cells and CK8+ luminal progenitor cells.

OLFM4 is highly co-expressed with stem/progenitor cell markers in RWPE1 cells. We have previously detected OLFM4 RNA and protein expression in RWPE1 cells ${ }^{35}$. RWPE1 cells are immortalized normal adult prostate epithelial cells whose growth can be maintained under serum-free conditions in 2D culture. We sought to identify OLFM4-expressing cells in the RWPE1 cell population through single-cell RNA sequencing of a total of 5000 single cells obtained from $2 \mathrm{D}$ culture. Thirteen clusters were identified by analyzing gene-expression signatures with Uniform Manifold Approximation and Projection (UMAP) software (Fig. 2a, left panel). High numbers of OLFM4-expressing cells were located in cluster 7, in which the stem/progenitor genes KRT13 and KRT19 were also expressed, and in cluster 3, in which the stem/progenitor genes LY6D and KLK11 were 
a
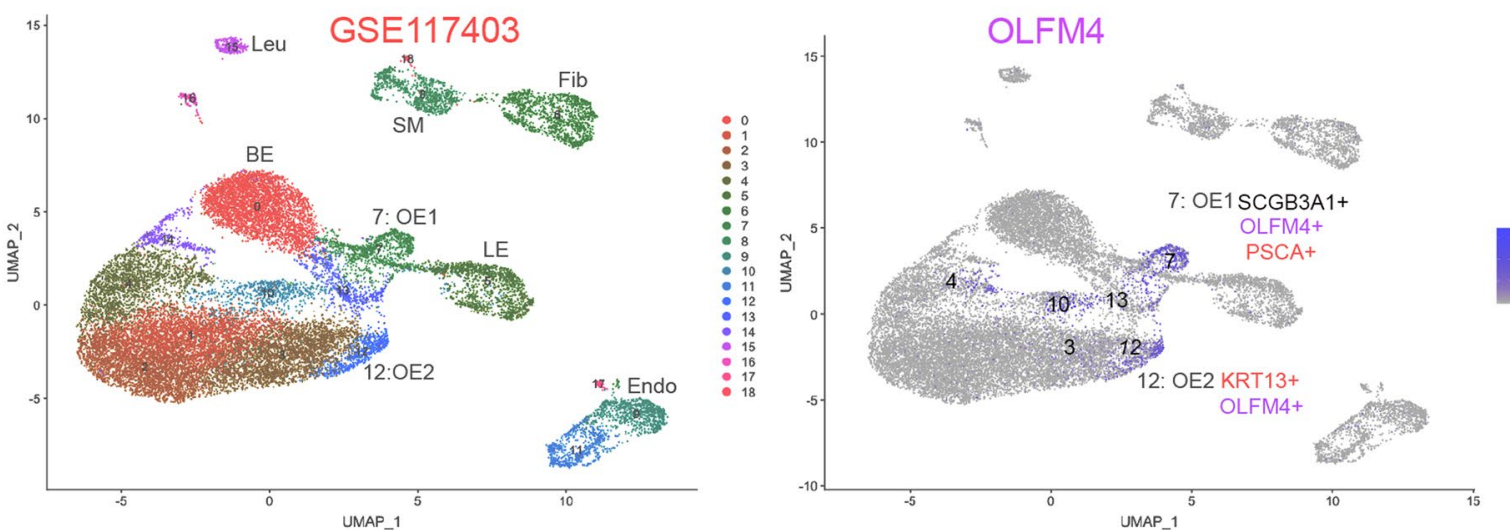

b
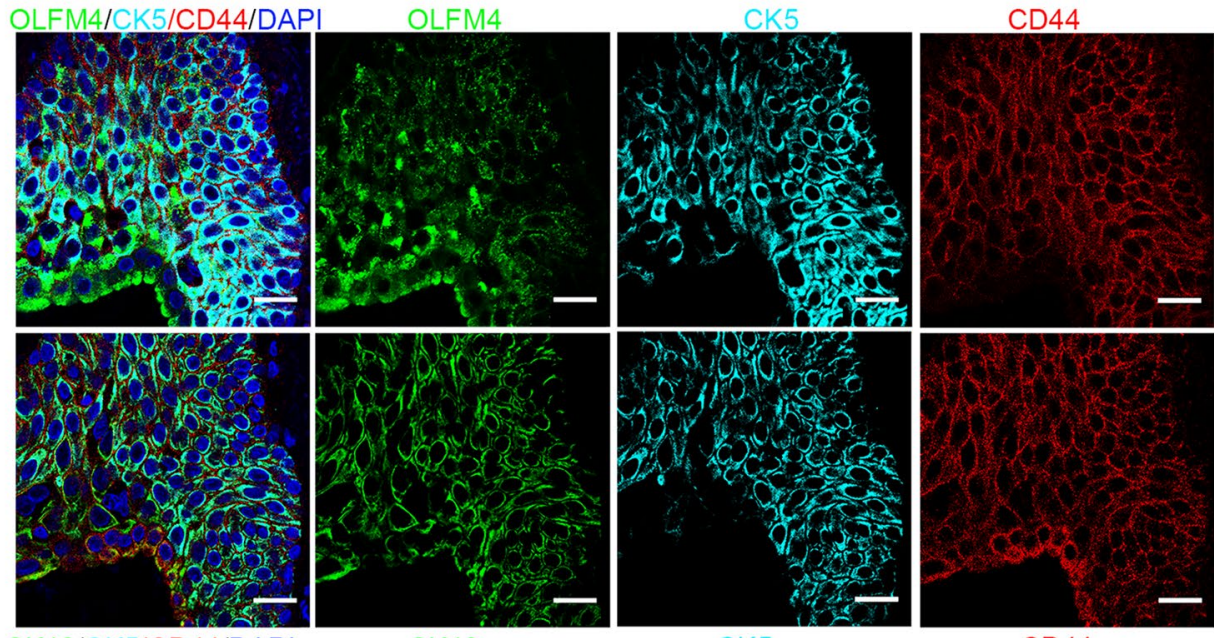

DAP
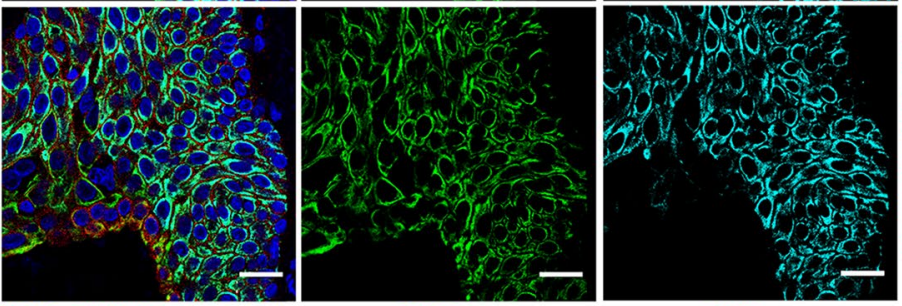

CK13/CK5/CD44/DAP

CK13

CK5

CD44

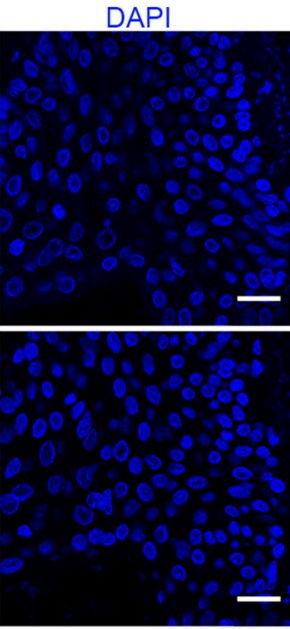

DAPI

C

OLFM4/SCGB1A1/DAPI

OLFM4/SCGB1A1/DAP
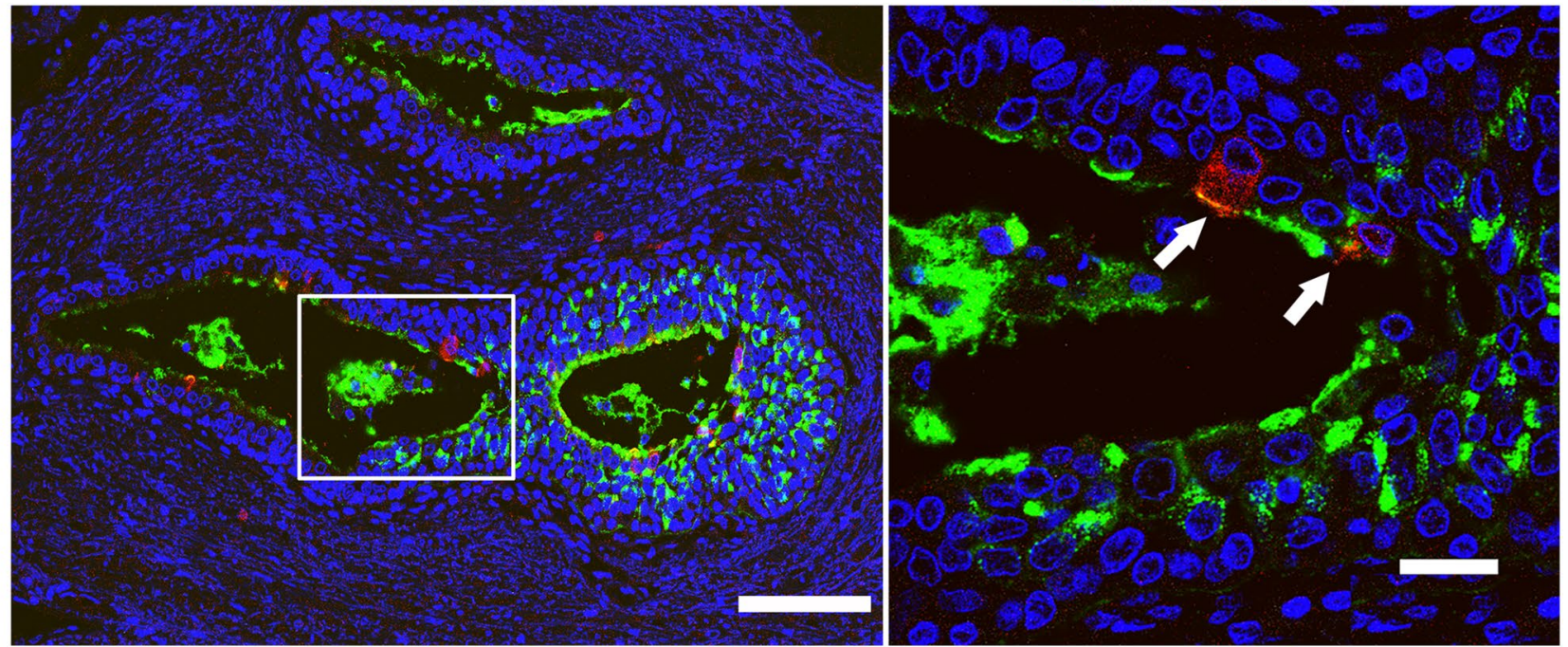

Figure 1. Identification of OLFM4-expressing cells in normal human adult prostate epithelium. (a) Uniform Manifold Approximation and Projection (UMAP) plots of integrated data from single-cell RNA sequencing of the GSE117403 dataset. Left panel shows 19 clusters of total prostate cells; right panel shows OLFM4-expressing cells (purple color) in clusters 7 and 12. (b) Representative triple-color immunofluorescent staining of normal prostatic urethra tubular epithelium. OLFM4 and CK13 (green); CD44 (red); CK5 (cyan); DAPI (blue). Scale bar: $20 \mu \mathrm{m}$. (c) Representative double-color immunofluorescent staining of normal prostatic tissues. OLFM4 (green); SCGB1A1 (red); DAPI (blue). Left panel shows low-magnification image. Scale bar: $100 \mu \mathrm{m}$. Right panel shows high-magnification image. Scale bar: $20 \mu \mathrm{m}$. Arrow indicates OLFM4+/SCGB1A1+ cells. 
a
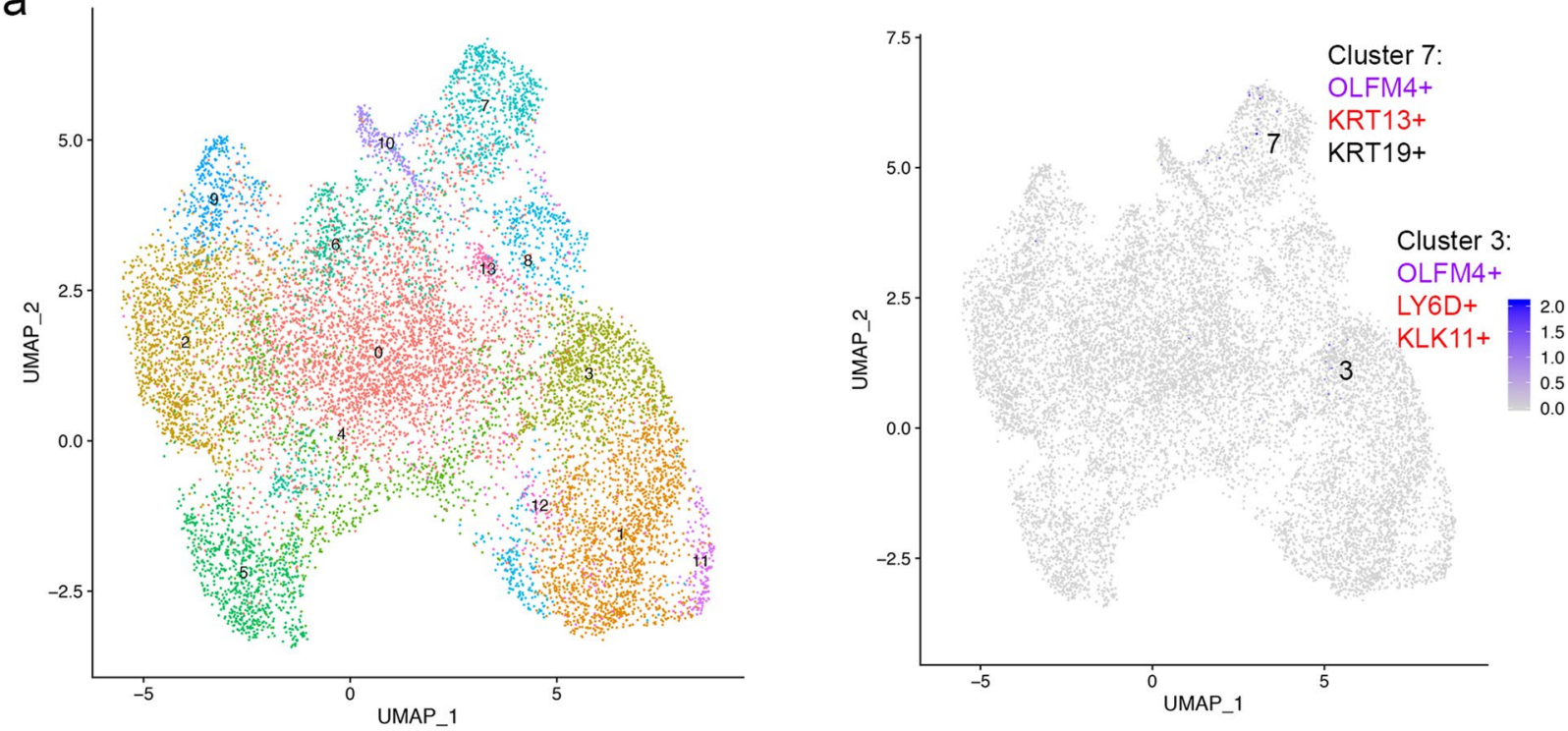

b

C
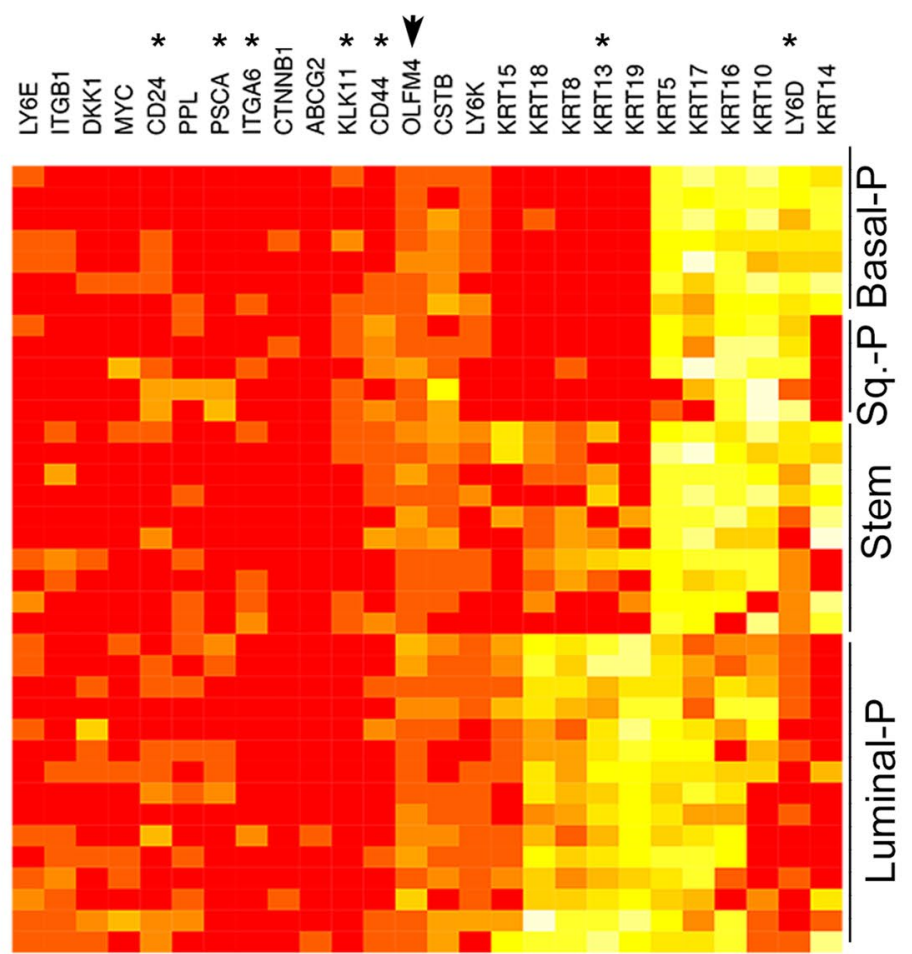

log2(normalizedExpression+1)
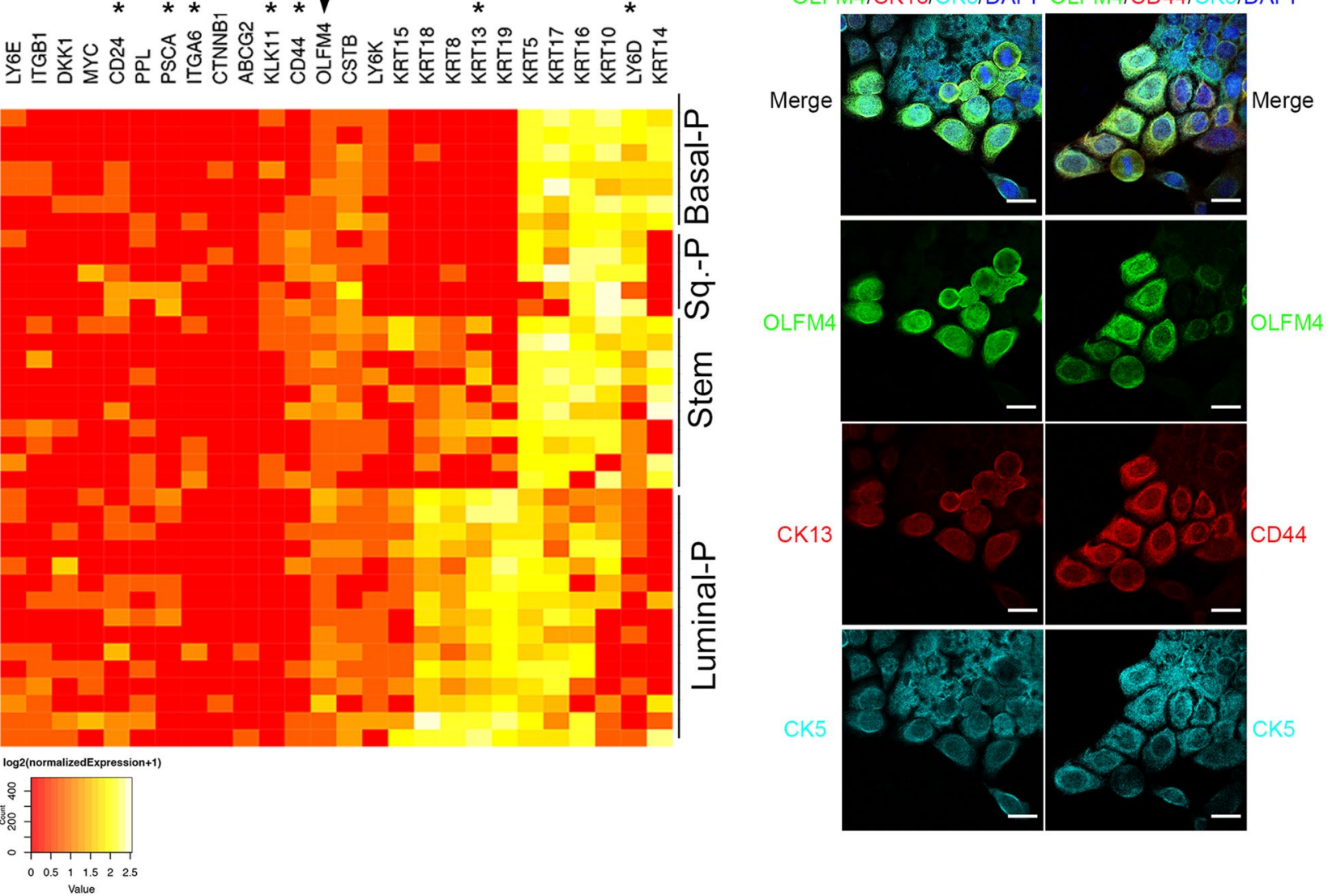

Figure 2. Identification of OLFM4-expressing RWPE1 cells. (a) Uniform Manifold Approximation and Projection (UMAP) plots of integrated data from single-cell RNA sequencing of RWPE1 cells. Left panel shows 13 clusters of total RWPE1 cells; right panel shows OLFM4-expressing cells (purple color) in clusters 3 and 7. (b) Heat map illustrates OLFM4 co-expression with stem/progenitor-cell marker genes, cytokeratins, and others in 37 OLFM4-expressing RWPE1 cells. Sq., squamous. P, progenitor. ${ }^{\star}$ Indicates lines for stem/progenitor-cell marker genes; arrow indicates line for the OLFM4 gene. (c) Representative triple-color immunofluorescent staining of RWPE1 cells. OLFM4 (green); CK13 and CD44 (red); CK5 (cyan); DAPI (blue). Scale bar: $20 \mu \mathrm{m}$. 
also expressed (Fig. 2a, right panel and Supplementary Fig. S3). The higher level of OLFM4-expressing cells distributed in the stem/progenitor-like cell populations was shown in a heat map generated from single-cell RNA sequencing of RWPE1 cells (Fig. 2b). We detected a 0.74\% OLFM4 RNA expression rate (that is, OLFM4 expression was observed in 37 cells from the total of 5000 single RWPE1 cells that were RNA sequenced). As shown in the heat map, the population of OLFM4-expressing cells that were stem-like cells was $27.0 \%$ (10 out of 37), that were basal progenitor-like cells was $18.9 \%$ (7 out of 37), that were luminal progenitor-like cells was $40.5 \%$ (15 out of 37), and that were squamous progenitor-like cells was 13.5\% (5 out of 37). Several cells expressed different combinations of stem/progenitor-cell marker genes, such as KRT13, LY6D, PSCA, CD44, ITGA6, and CD24. Further analysis of stem/progenitor cells found that 78.4\% of OLFM4+ cells were LY6D+ (29 out of 37), $54.1 \%$ of OLFM4+ cells were KRT13+ (20 out of 37$), 45.9 \%$ of OLFM4+ cells were CD44+ (17 out of 37), 43.2\% of OLFM4+ cells were CD24+ (16 out of 37$), 18.9 \%$ of OLFM4+ cells were PSCA+ (7 out of 37 ), and $18.9 \%$ of OLFM4+ cells were ITGA6+ (7 out of 37). The results suggest that multiple stem/progenitor cell populations exist in RWPE1 cells and OLFM4 was more frequently co-expressed with LY6D and KRT13 stem-cell markers.

Examination of RWPE1 cells with triple-color immunofluorescent staining demonstrated that OLFM4 was co-expressed with CK13, CD44, CK5 and SCGB1A1 (Fig. 2c, Supplementary Fig. S3). We further observed that OLFM4-positive cells co-expressed with CK8 cell markers (Supplementary Fig. S3). OLFM4-positive cells did not express P63, AR, and synaptophysin markers (Supplementary Fig. S3). These results verified single-cell RNA sequencing data indicating that OLFM4 is expressed in multiple stem/progenitor-like cell populations in RWPE1 cells.

OLFM4-knockout RWPE1 cells are enriched in CD49F+ and CD44+ stem/progenitor-like cell populations. Our earlier data demonstrated that OLFM4 was expressed in multiple stem/progenitor cell types within the wild-type OLFM4 RWPE1 cell population. To study OLFM4 gene function in human prostate stem/progenitor-like cells, we used CRISPR/Cas9 technology to establish OLFM4-knockout and OLFM4-wild RWPE1 cell clones that express green fluorescent protein (GFP). The cell clones were verified with genomic PCR sequencing and RT-PCR (Supplementary Fig. S4). We then performed FACS analysis using anti-GFP antibody combined with phycoerythrin (PE)-labeled antibodies to the cell-surface stem/progenitor-cell markers CD49F, CD44, CD26, or CD24. GFP+/CD49F+ cells and GFP+/CD44+ cells were highly enriched in OLFM4-knockout GFP reporter RWPE1 cells compared with OLFM4-wild GFP reporter RWPE1 cells (Fig. 3a, left two columns of panels). In contrast, GFP+/CD26+ cells and GFP+/CD24+ cells were not enriched in OLFM4-knockout GFP reporter RWPE1 cells compared with OLFM4-wild GFP reporter RWPE1 cells (Fig. 3a, right two columns of panels). These results indicate that OLFM4 knockout enriched CD49F+ and CD44+ cell populations in RWPE1 cells.

OLFM4-knockout RWPE1 stem/progenitor-like cells exhibit enhanced growth via symmetric division in 2D culture. To identify individual RWPE1 stem/progenitor-like cells, we performed time course single-GFP+ cell tracing on 2D cultures of OLFM4-wild and OLFM4-knockout GFP reporter RWPE1 cells that were generated by transfecting the CRISPR/Cas 9 OLFM4 activation or knock out plasmids into the RWPE1 OLFM4-wild cells (Supplementary Fig. S4). We found that OLFM4-knockout stem/progenitor-like cells proliferated by undergoing both symmetric division (self-renewal) and asymmetric division (giving rise to one stem cell and one progenitor cell) at day 4 to day 7 (Fig. 3b,c). We also found symmetric commitment OLFM4-knockout GFP reporter RWPE1 cells (Fig. 3d). In contrast, we observed that OLFM4-wild GFP reporter RWPE1 cells stayed in quiescence and slowly asymmetric divided at day 4 to day 7 (Fig. 3b and Supplementary Fig. S4). To quantify the number of symmetric and asymmetric cells produced by each GFP reporter cell line, the GFP-positive cells at day 4 of 2D culture were counted. At that timepoint, 20.1\% (44/219) of OLFM4-wild GFP reporter RWPE1 cells were in symmetric division and 79.9\% (175/219) were in asymmetric division (Fig. 3e). In contrast, $70.6 \%(204 / 289)$ of OLFM4-knockout GFP reporter RWPE1 cells were in symmetric division and $29.4 \%(85 / 289)$ were in asymmetric division. These results suggest that OLFM4 promotes stem/progenitor-like cell asymmetric division, whereas OLFM4 knockout shifts stem/progenitor-like cell division to favor symmetric division.

OLFM4-GFP reporter RWPE1 stem/progenitor-like cells proliferate and differentiate in 3D Matrigel culture. Matrigel 3D sphere formation assays and organoid assays has previously been used to evaluate the self-renewal and differentiation potential of stem/progenitor cells ${ }^{19,38,39}$. To identify properties of OLFM4-expressing RWPE1 stem/progenitor-like cells, OLFM4-knockout or OLFM4-wild GFP reporter RWPE1 cells were analyzed in 3D Matrigel culture using prostate sphere-formation assays and organoid assays from single-GFP+ cells. We observed single-GFP+ cells to follow sphere formation from day 1 to day 14 under sphere culture conditions (Fig. 4a). We observed three growth patterns: spheres within the Matrigel; colonies attached to the surface of plates; and branches within the Matrigel in the sphere formation assays (Fig. 4b). OLFM4knockout GFP reporter RWPE1 cells formed significantly more spheres and colonies but fewer branches than OLFM4-wild GFP reporter RWPE1 cells (Fig. 4b). The cell populations found within the spheres were identified by immunohistochemical staining (Supplementary Fig. S5). All OLFM4-knockout RWPE1 sphere formed cells expressing CD44 stem/progenitor cell marker and CK5/CK14 basal cell markers. Most of cells expressing epithelial marker, E-cadherin and some cells expressing mesenchymal cell marker, vimentin. We did not detect differentiated luminal cell markers CK8 and AR as well as epithelial-mesenchymal transition marker, $\mathrm{N}$-cadherin expression in those cells (Supplementary Fig. S5).

To further identify differentiation abilities of OLFM4-expressing RWPE1 stem/progenitor-like cells, we performed organoid culture assays with OLFM4-wild and OLFM4-knockout GFP reporter RWPE1 cells. We traced 
a

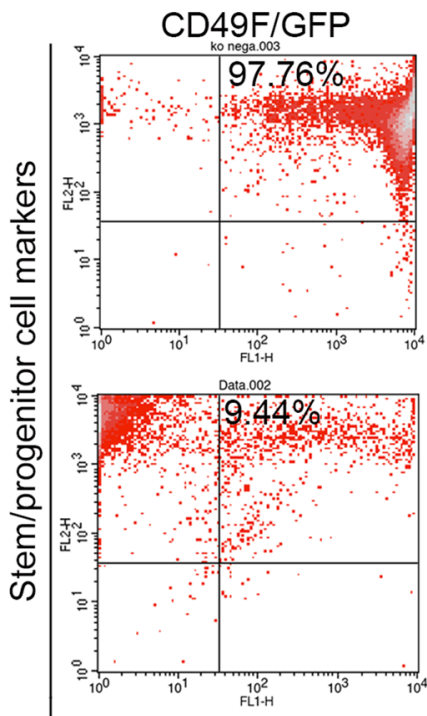

b

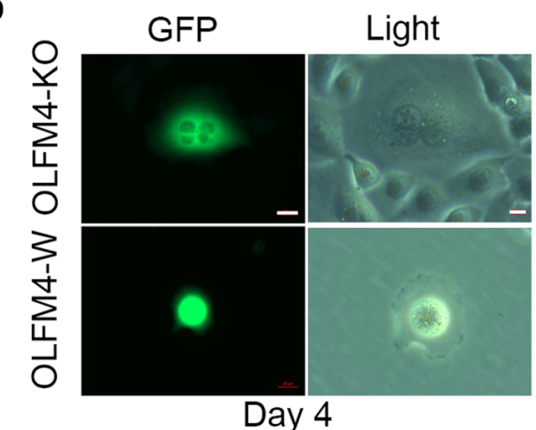

d

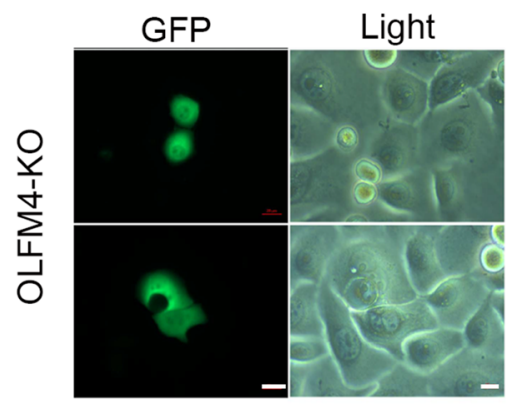

Day 4
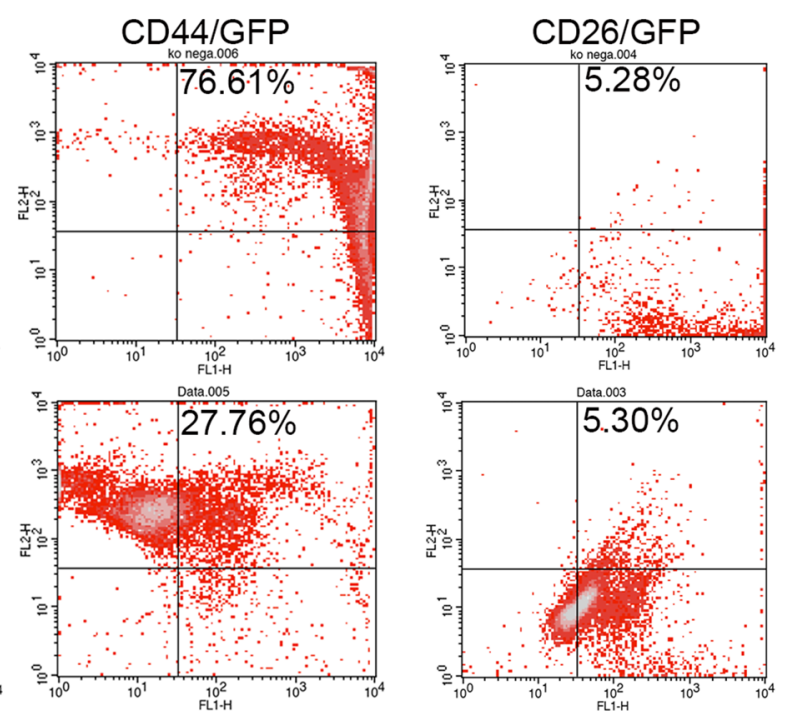

C

Light
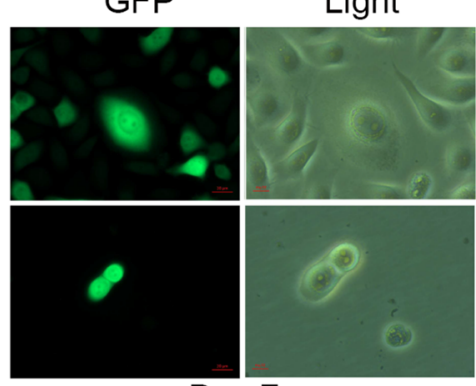

Day 7

e

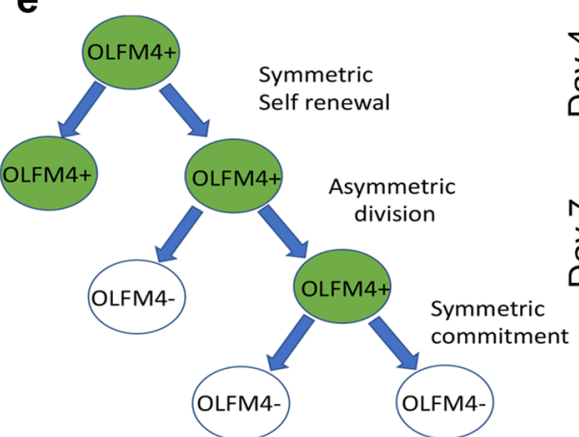

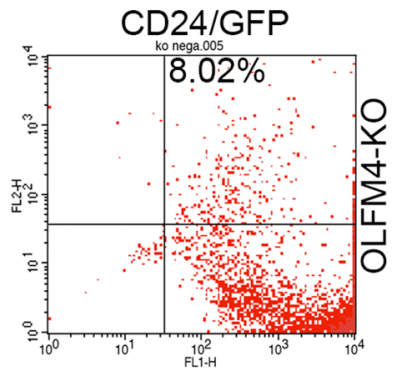
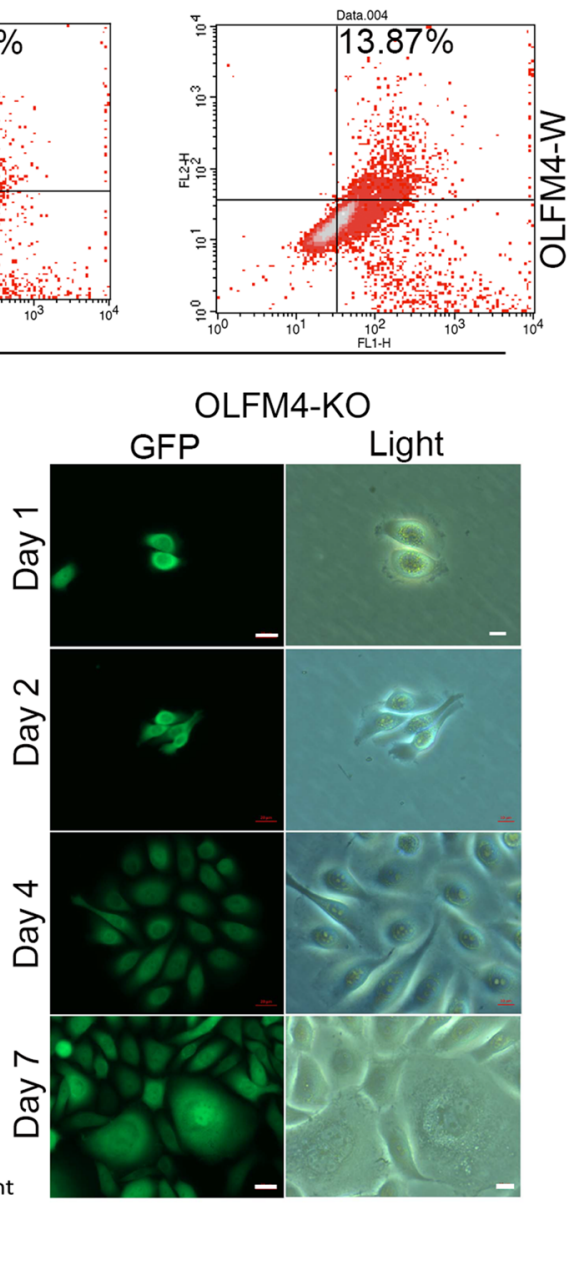

Figure 3. Characterization of OLFM4-knockout GFP reporter RWPE1 cells in 2D culture. (a) Representative FACS analysis of OLFM4-wild and OLFM4-knockout GFP reporter RWPE1 cells using antibodies to GFP combined with antibodies to stem/progenitor-cell markers CD49F, CD44, CD26, or CD24. Inset values indicate the percentage of double-marker-positive cell populations. (b) Representative GFP- and light-field images generated using single-cell tracing of OLFM4-wild and OLFM4-knockout GFP reporter RWPE1 cells at 4 and 7 days in 2D culture. Scale bar: $10 \mu \mathrm{m}$. (c) Representative GFP- and light-field images of OLFM4-knockout GFP reporter RWPE1 cells at 1,2, 4, and 7 days in 2D culture. Scale bar: $10 \mu \mathrm{m}$. (d) Representative GFP- and light-field images of OLFM4-knockout GFP reporter RWPE1 cells at 4 days in 2D culture. Scale bar: $10 \mu \mathrm{m}$. (e) Illustration of OLFM4-expressing GFP-positive RWPE1 stem/progenitor-like cell symmetric and asymmetric division. OLFM4-KO, knockout; OLFM4-W, wild.

single cells using the GFP reporter protein to follow organoid formation from day 1 to day 12 under organoid culture conditions (Fig. 4c). Both large and small organoids with compact cells exhibiting basal stem/progenitor cell-like morphology were observed after 12 days of culture (Fig. $4 \mathrm{~d})^{17}$. We performed double-color immunofluorescent staining of organoids after 12 days of culture for identifying cell populations within the large and small organoids. We detected the expression of luminal cell markers (CK8, AR), basal cell markers (CK5, CK14, and P63), and stem/progenitor cell markers (CK19 and CK13) in the large organoids obtained from both OLFM4wild and OLFM4-knockout GFP reporter RWPE1 cells (Fig. 4e). Interestingly, we observed a few cells presented 
a

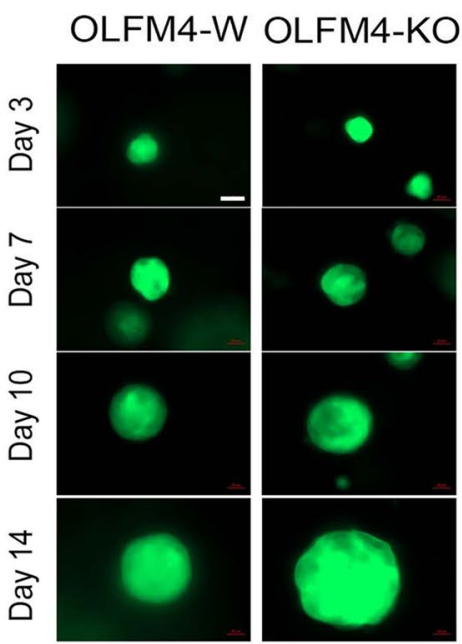

C

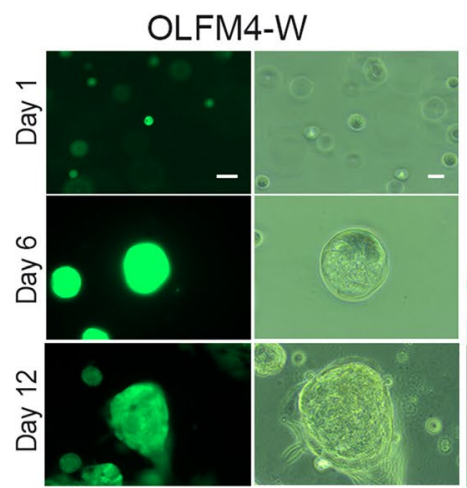

b
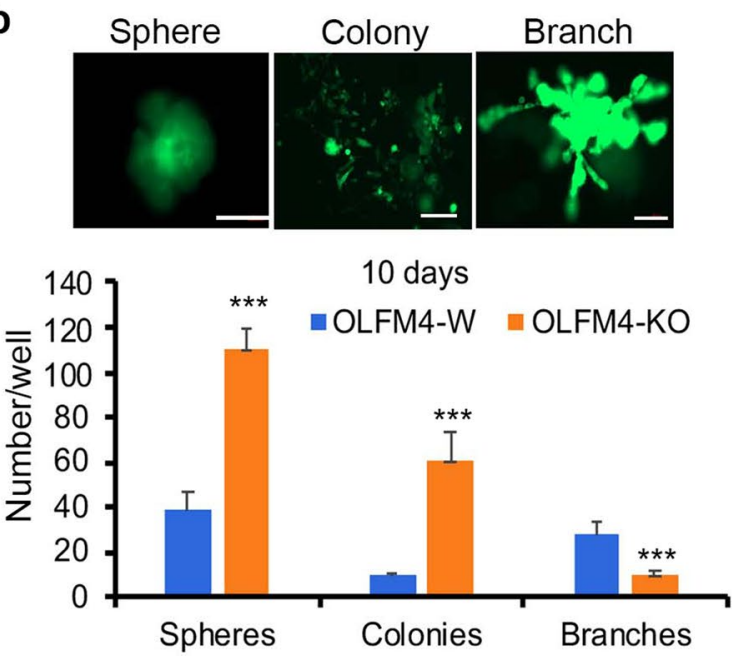

d e
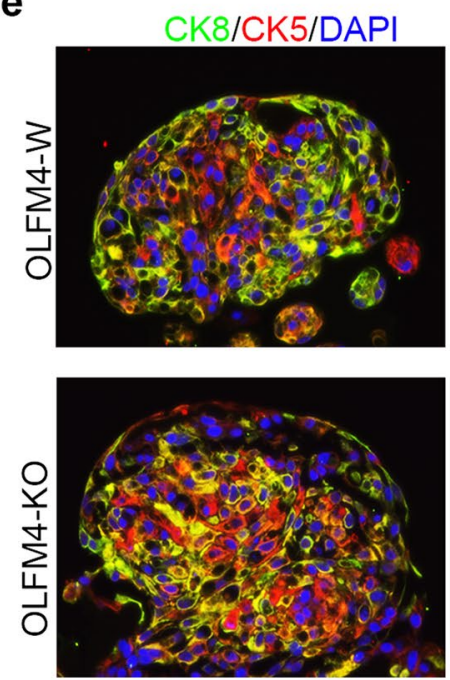
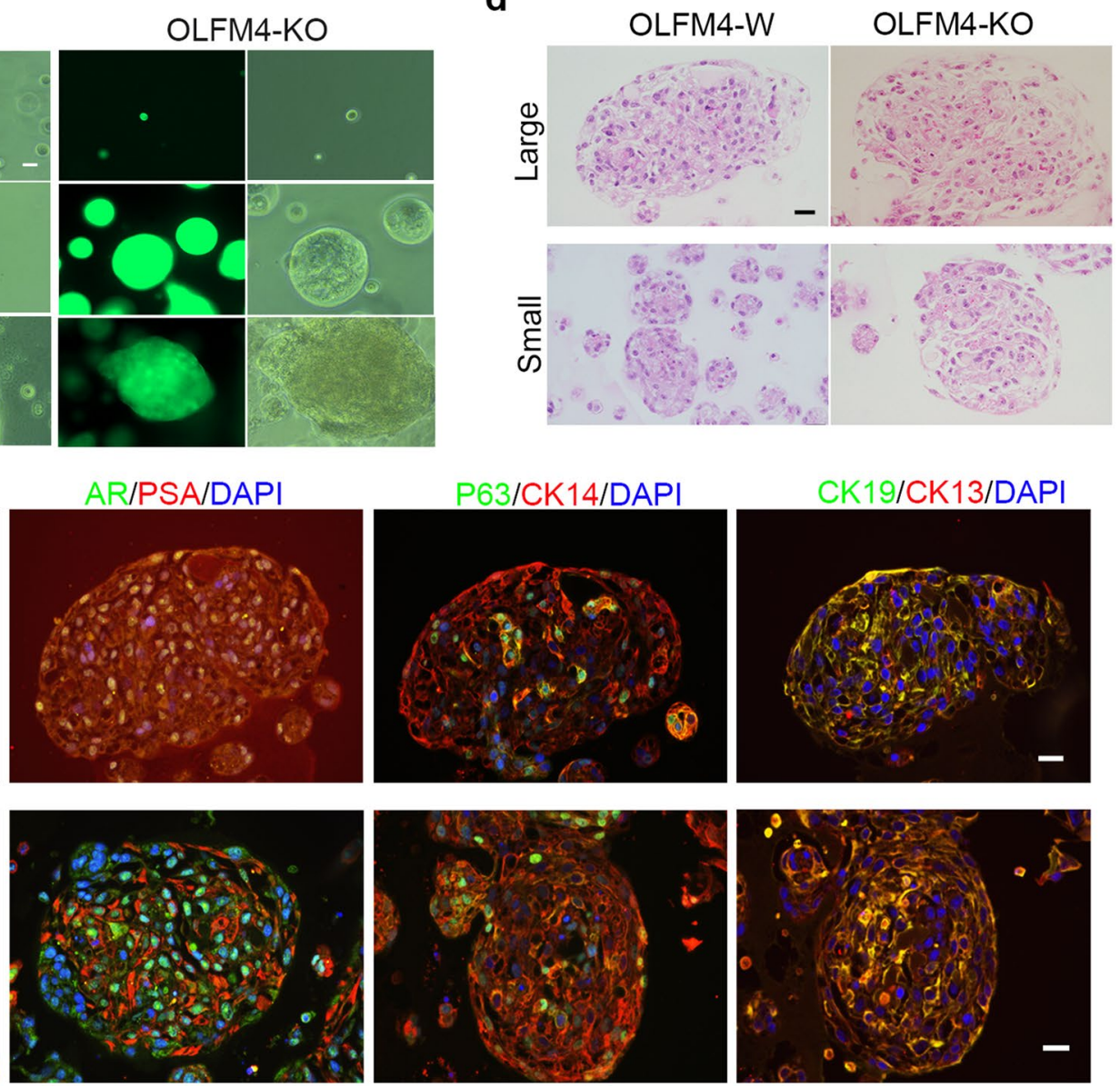

Figure 4. Characterization of OLFM4-GFP reporter RWPE1 cells in 3D Matrigel culture. Prostate sphereformation and organoids assays were performed with OLFM4-wild and OLFM4-knockout GFP reporter RWPE1 cells grown in Matrigel. (a) Representative GFP-field sphere images using single-cell tracing over time (scale bar: $20 \mu \mathrm{m}$ ). (b) Representative GFP-field images of sphere, colony and branch formation after 10 days in Matrigel culture. Scale bar: $100 \mu \mathrm{m}$. Bar graph presents mean number ( \pm standard deviation; SD, n=6) of spheres ( $>50 \mu \mathrm{m}$ in diameter), colonies, or branches formed after 10 days in culture. ${ }^{* * *} p<0.001$ when compared with OLFM4-wild RWPE1 cells (Student's t-test). (c) Representative GFP- and light-field images generated using single-cell tracing of OLFM4-wild (W) and OLFM4-knockout (KO) GFP reporter RWPE1 cells grown to organoids at 1, 6, and 12 days in culture. Scale bar: $20 \mu \mathrm{m}$ (GFP) or $10 \mu \mathrm{m}$ (light). (d) Representative hematoxylin-eosin staining of organoids at 12 days in culture. Scale bar: $20 \mu \mathrm{m}$. (e) Representative double-color immunofluorescent staining of large organoids at 12 days in culture. DAPI (blue) was used for nuclei staining. Scale bars: $20 \mu \mathrm{m}$. OLFM4-W, OLFM4-wild; OLFM4-KO, OLFM4-knockout. 
lower level PSA expression in the large organoids from OLFM4-wild GFP reporter RWPE1 cells. In contrast, we observed more than half cells presented higher level PSA expression in the large and small organoids from OLFM4-knock out GFP reporter RWPE1 cells (Fig. 4e and Supplementary Fig. S5).

Taken together, these data suggest that OLFM4-knockout RWPE1 cells exhibit higher proliferative abilities and differentiated into higher levels PSA expression cells when compared with OLFM4-wild RWPE1 cells.

OLFM4-knockout RWPE1 cells exhibit enhanced MYC-signaling target gene signatures. To explore the molecular mechanisms underlying OLFM4 mediation of RWPE1 cell self-renewal and differentiation, we performed bulk-cell RNA sequencing analysis (Fig. 5). OLFM4-knockout RWPE1 cells were sorted by GFP-marker expression, then bulk-cell RNA sequencings were performed (Fig. 5a). We first analyzed and performed quantitative real-time RT-PCR for picked genes. We found expression of stem-cell marker genes KRT13, $L Y 6 D, K L K 10$, and ITGA6 were enhanced, but the luminal progenitor cell marker genes CD24 and PSCA were reduced in OLFM4-knockout GFP reporter RWPE1 cells compared with OLFM4-wild RWPE1 cells (Supplementary Fig. S6). The $M Y C$ gene was significantly increased, while in contrast other transcription factors, such as prostate specific transcription factor, $H O X B 13, N K X 3.1$, and BMI1, were reduced in OLFM4-knockout GFP reporter RWPE1 cells compared with OLFM4-wild RWPE1 cells (Supplementary Fig. S6). The RNA expression of basal cell marker genes, KRT5 and KRT14, were increased (Supplementary Fig. S6) but luminal cell marker genes KRT8 and KRT18 were reduced (Supplementary Fig. S6) in OLFM4-knockout GFP reporter RWPE1 cells compared with OLFM4-wild RWPE1 cells. These results were consistent with FACS data that OLFM4 knockout enriched more basal stem/progenitor-like cells, which highly express MYC, in RWPE1 cells.

We further identified genes from whole-genome transcriptome analysis (22,339 genes) with a greater than twofold Log FC change when OLFM4-knockout GFP reporter RWPE1 cells were compared with OLFM4-wild RWPE1 cells (Log FC OLFM4-KO/OLFM4-W; Supplementary Table S2). This analysis revealed 199 upregulated genes and 1443 downregulated genes. Gene set enrichment analysis (GSEA) revealed an enrichment pattern of gene signatures related to stem cells, such as mammary stem-cell upregulated genes, as well as an embryonic stem-cell signature in OLFM4-knockout GFP reporter RWPE1 cells (Fig. 5b). These enriched stem-cell signaling pathway signatures were pinpointed to be for WNT-signaling and APC/MYC-signaling target genes (Fig. 5c and Supplementary Fig. S7) $)^{40}$. We verified increased MYC gene expression in OLFM4-knockout GFP reporter RWPE1 cells using qRT-PCR, Western blot analysis, and immunofluorescent staining (Supplementary Fig. S7). The MYC target genes signature was found to be enriched in OLFM4-knockout GFP reporter RWPE1 cells in data obtained from three different data resources (Fig. 5d and Supplementary Fig. S7). To test function of the MYC gene in RWPE1 cells, we used (+)-JQ1, a MYC inhibitor, in both 2D and 3D culture models, and found that (+)-JQ1 substantially inhibited proliferation of OLFM4-knockout GFP reporter RWPE1 cells compared with OLFM4-W RWPE1 cells in both types of cultures (Fig. 5e-g). These results provided further evidence that OLFM4 mediates RWPE1 cell proliferative processes through MYC signaling pathways.

RNA-sequencing data analysis demonstrates potential MYC-related molecular mechanisms of the OLFM4 gene in RWPE1 stem/progenitor-like cells. We further analyzed RNA sequencing data to identify gene ontology enrichments in OLFM4-knockout GFP reporter RWPE1 cells compared with OLFM4-wild RWPE1 cells. This analysis yielded 5 positively enriched and 12 negatively enriched gene signatures for biological processes (Fig. 6a), 9 positively enriched and 1 negatively enriched gene signature for cellular components (Fig. 6b), and 9 positively enriched gene signatures for molecular functions (Fig. 6c). Further analysis from gene ontology demonstrated significantly enriched reactomes including metabolism of RNA, translation, peptide chain elongation, and respiratory electron transport (Fig. 7a). The pathways analysis revealed 10 significantly enriched pathways, such as Electron transport chain (OXPHOS system in mitochondria) from Wikipathways pathways (Fig. 7b), Ribosome biogenesis in eukaryotes from KEGG pathways (Fig. 7c), and cell cycle from Panther pathways (Fig. 7d). These results suggest that OLFM4 negatively mediates MYC protein functions related to cell proliferation, metabolism, ribosome biogenesis, protein synthesis, and mitochondrial function.

\section{Discussion}

We report for the first time that OLFM4-expressing cells represent multiple stem/progenitor-like cell populations and that the OLFM4 gene plays an important role in cell self-renewal and differentiation. Therefore, the OLFM4 gene might be useful for lineage tracing of normal prostate stem/progenitor cells during organogenesis and homeostasis of prostate.

Prostate stem/progenitor cells have been identified in the urogenital sinus epithelium, prostatic buds, and solid prostatic tube during prostate organogenesis, as well as in the adult prostate urethra tube epithelium and prostate grands ${ }^{41,42}$. Recently, Henry et al. reported two clusters of stem/progenitor cells in the normal adult prostate epithelium based on their gene expression signature obtained from scRNA sequencing, classifying them as KRT13+Hillock and SCGB1A1+Club cells ${ }^{8}$. Because their scRNA sequencing data are publicly available in the GEO database, we performed bioinformatic analysis on those data and found higher OLFM4 expression in cluster 7 (OLFM4+/SCGB3A1+/PSCA+/CD24+) and in cluster 12 (OLFM4+/KRT13+/KRT19+) prostate stem/ progenitor cells in normal adult prostate. Due to tissue resource limitations, we used the immortalized human normal adult prostate epithelial cell line, RWPE1, for further studies of OLFM4-expressing stem/progenitor-like cell populations. The gene-expression signature of RWPE1 stem/progenitor cells has been shown to conserve most genes in the normal and benign prostate stem/progenitor cell signature ${ }^{8,9}$. Therefore, we used these cells to mimic stem/progenitor cell self-renewal and differentiation in the organogenesis of prostate epithelium. 
a

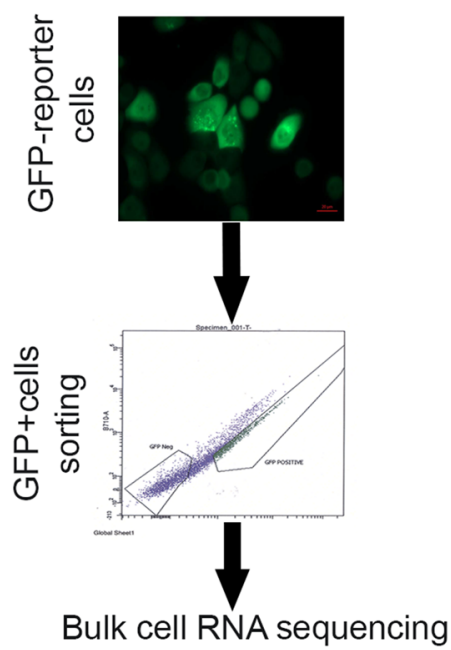

d

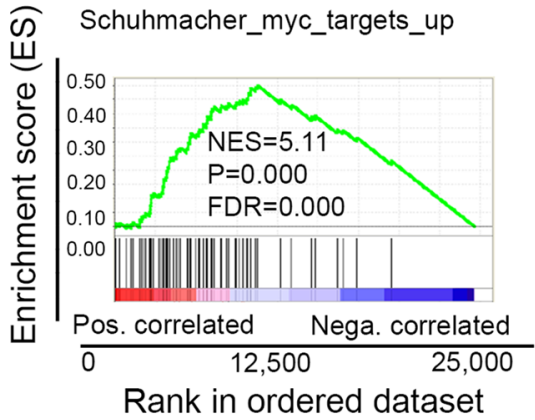

b

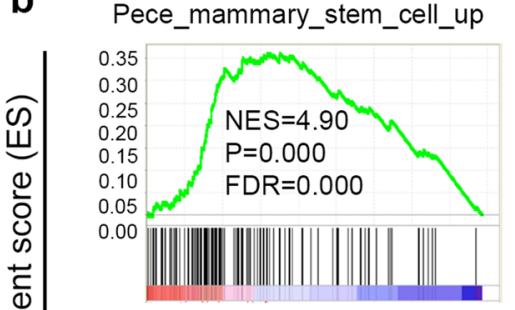

Wong_embryonic_stem_cell_core

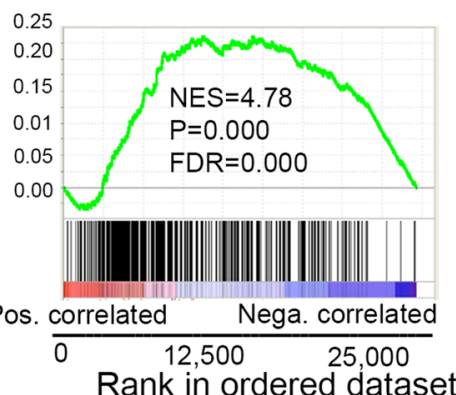

Dang_regulated_by_myc_up

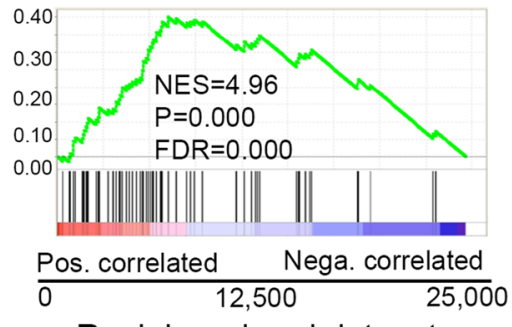

Rank in ordered dataset
C

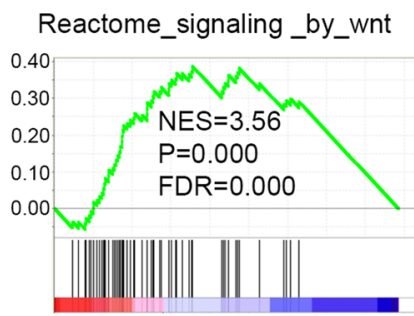

Sanson_apc_targets_require_myc

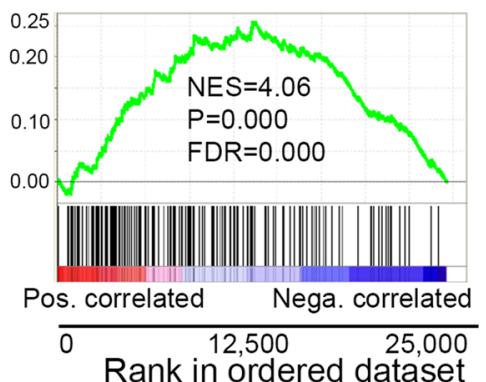

Menssen_myc_targets

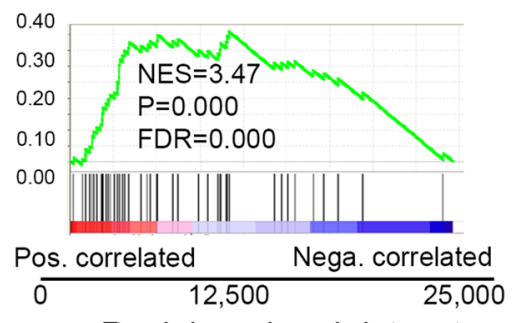

Rank in ordered dataset e

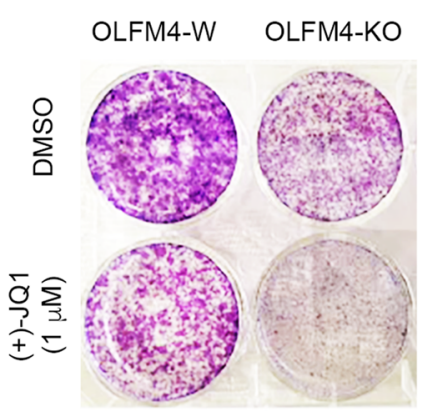

f

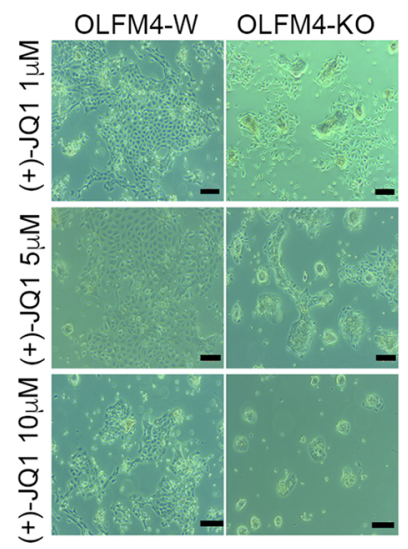

g

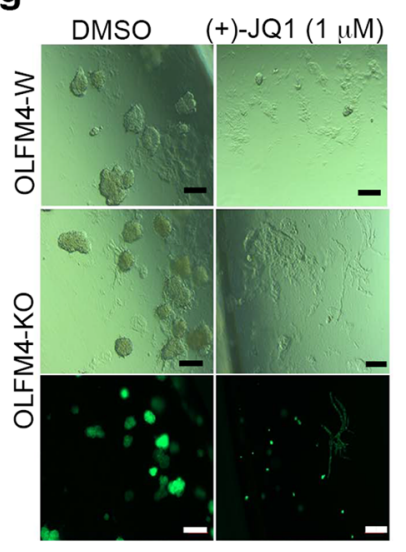

Figure 5. GSEA analysis for OLFM4-knockout GFP reporter RWPE1 cells from bulk-cell RNA sequencing data. (a) Strategy of bulk-cell RNA sequencing for FACS cell sorting by GFP-marker expression of OLFM4-GFP reporter RWPE1 cells. (b) GSEA showing enrichment of stem-cell-like gene signatures in OLFM4-knockout GFP reporter RWPE1 cells. (c) GSEA showing enrichment of WNT-signaling and APC/MYC-signaling target gene signatures in OLFM4-knockout GFP reporter RWPE1 cells. (d) GSEA showing enrichment of MYC target gene signatures in OLFM4-knockout GFP reporter RWPE1 cells from three different data resources. (e) Representative images of colonies of OLFM4-wild and OLFM4-knockout GFP reporter RWPE1 cells cultured in the presence of $1 \mu \mathrm{M}(+)$-JQ1 (or DMSO, vehicle control) for 7 days in 6-well plates, then stained with the Diff-Quik Stain Set. (f) Representative images of colonies of OLFM4-wild and OLFM4-knockout GFP reporter RWPE1 cells cultured in the presence of $1 \mu \mathrm{M}, 5 \mu \mathrm{M}$, or $10 \mu \mathrm{M}$ (+)-JQ1 (or DMSO, vehicle control) for 7 days. Scale bar: $100 \mu \mathrm{m}$. (g) Representative GFP- and light-field images of spheres of OLFM4-wild and OLFM4-knockout GFP reporter RWPE1 cells cultured in the presence of $1 \mu \mathrm{M}(+)-J Q 1$ (or DMSO, vehicle control) for 10 days in 3D culture. Scale bar: $100 \mu \mathrm{m}$. Bar graph presents mean number of spheres $( \pm S D, n=6)$ from these experiments using $100 \mathrm{nM}$ of (+)-JQ1 (or DMSO, vehicle control). W, wild; KO, knockout. ${ }^{* *} p<0.001$ (Student's t-test). 
a

NADH dehydrogenase complex assembly mitochodrial respiratory chain complex assembly mitochondrial gene expression rRNA metabolic process tRNA metabolic process

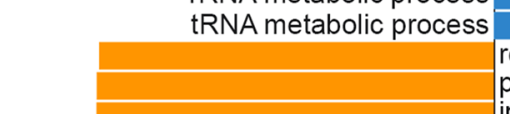

response to interferon-alpha

positive chemotaxis

interleukin-1 production

foam cell differentiation

humoral immune response

negative chemotaxis

response to interferon-beta

detection of biotic stimulus

response to tumor cell

response to fungus

response to type 1 interferon

peptide cross-linking

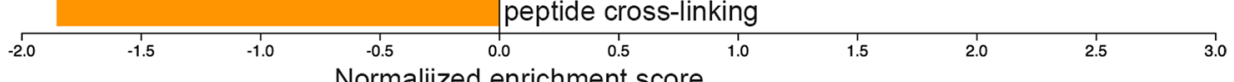

$\mathrm{b}$

$\mathrm{FDR} \leq 0.05$

\section{Cellular components}

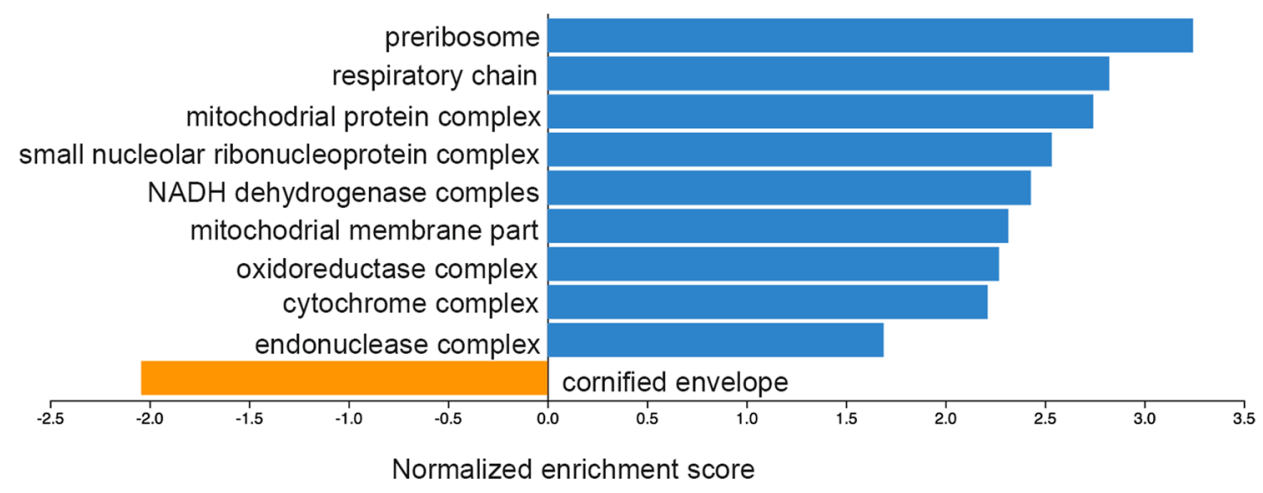

Normalized enrichment score

C

\section{Molecular functions}

FDR $\leq 0.05$

Threonine-type peptidase activity snoRNA binding

oxidoreductase activity, acting on a heme group of donors structural constituent of ribosome heme-copper terminal oxidase activity electron transfer activity macromolecule transmembrane transporter activity oxidoreductase activity, acting on $\mathrm{NAD}(\mathrm{P}) \mathrm{H}$ rRNA binding

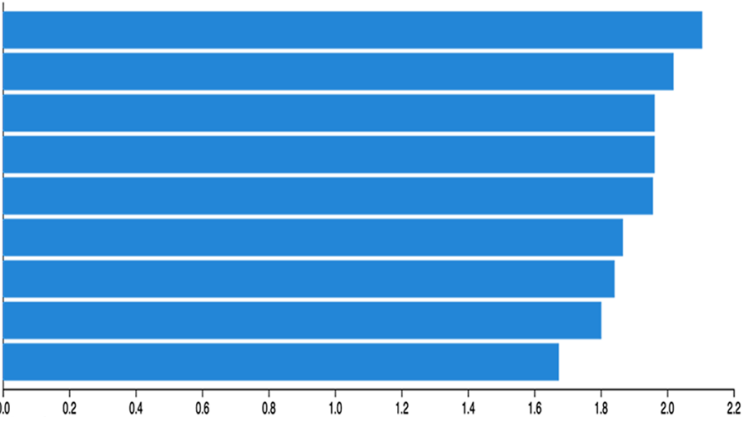

Normalized enrichment score

Figure 6. Gene ontology enrichments for OLFM4-knockout GFP reporter RWPE1 cells. (a) Bar graph presents upregulated (blue; FDR $\leq 0.05$ ) and downregulated (orange; FDR $\leq 0.05$ ) pathways for biological processes using WebGestalt 2019. (b) Bar graph presents upregulated (blue; FDR $\leq 0.05$ ) and downregulated (orange; FDR $\leq 0.05$ ) pathways for cellular components. (c) Bar graph presents upregulated (blue; FDR $\leq 0.05$ ) pathways for molecular functions.

Our data indicate that OLFM4 is a marker for multiple stem/progenitor-like cell populations within the RWPE1 cell population. In OLFM4-expressing stem/progenitor-like RWPE1 cell populations, we identified several stem/progenitor-cell marker genes, such as LY6D, KRT13, CD44, CD24, ITGA6, and PSCA. In particular, OLFM4 most frequently co-expressed with $L Y 6 D$, a marker for prostate stem cells that are castration resistant and an origin for prostate cancer ${ }^{43}$. More than half of the OLFM4-expressing RWPE1 cells expressed KRT13, which is highly expressed in Hillock cells localized in the prostatic urethra tube epithelium in what are called 
a

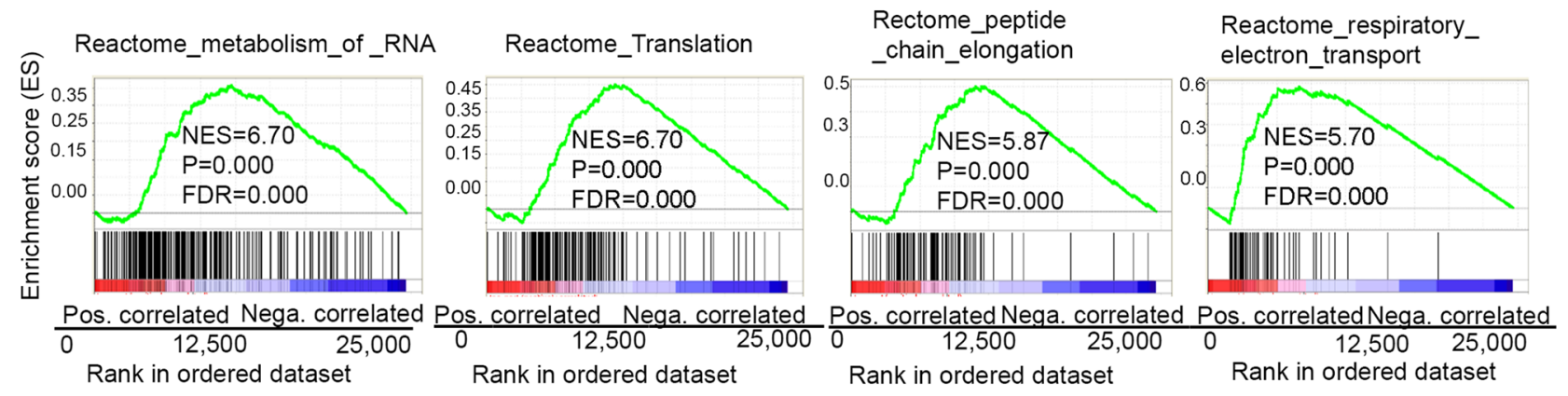

b

Pathway_wikipathways

FDR $\leq 0.05$

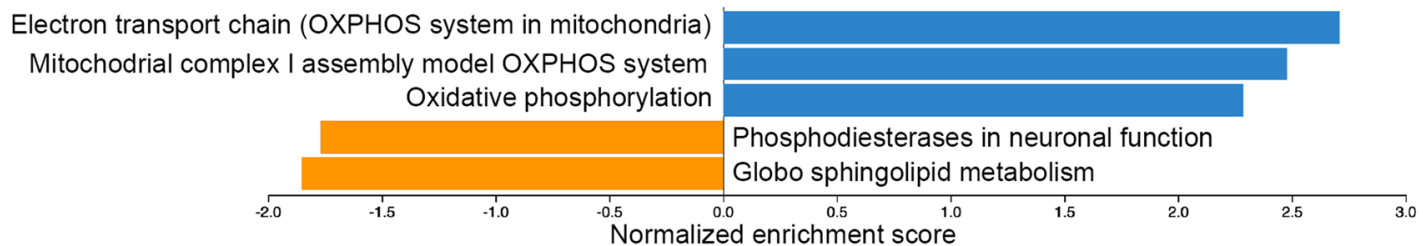

C

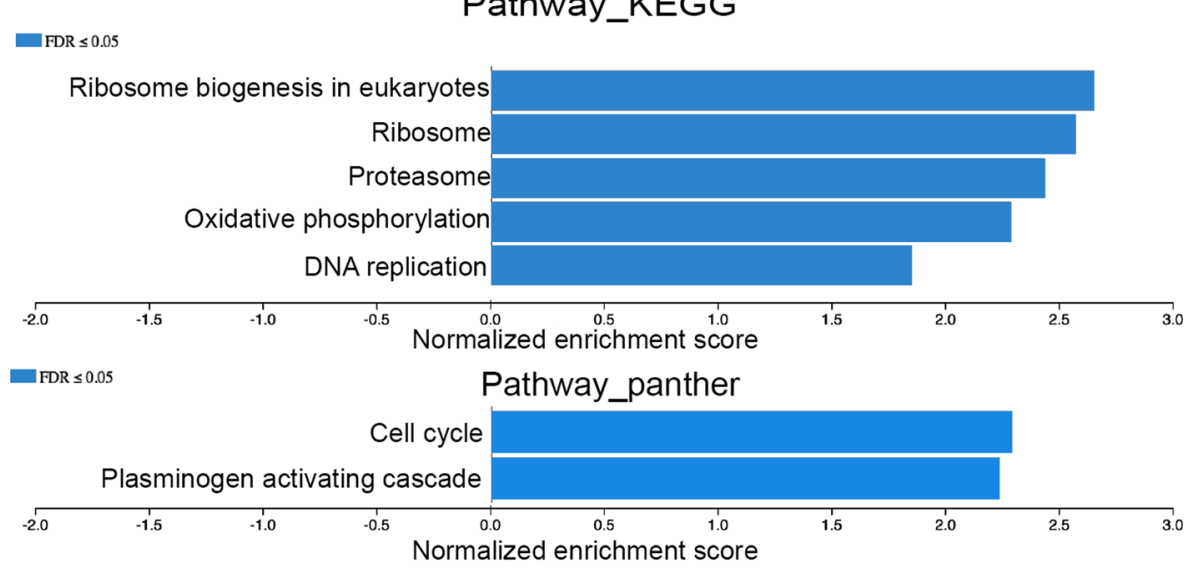

Figure 7. Reactomes and pathways enrichments for OLFM4-knockout GFP reporter RWPE1 cells. (a) Enrichment of reactomes in OLFM4-knockout GFP reporter RWPE1 cells. (b) Bar graph presents upregulated (blue; FDR $\leq 0.05$ ) and downregulated (orange; FDR $\leq 0.05$ ) pathways from Wikipathways. (c) Bar graph presents upregulated (blue; FDR $\leq$ 0.05) pathways from Pathway_KEGG. (d) Bar graph presents upregulated (blue; FDR $\leq 0.05$ ) pathways from Pathway_Panther.

prostatic stem cell niches in normal human prostate ${ }^{8,44}$. Almost half of the OLFM4+ RWPE1 cell population also expressed CD24, which is a luminal stem/progenitor cell marker ${ }^{45}$. The luminal progenitor cells have recently been identified as having an underlying role in prostate development, androgen-mediated regeneration of postcastration prostate, and the origin of prostate adenocarcinoma ${ }^{17,46-48}$. Therefore, our identification of multiple OLFM4-expressing stem/progenitor-like cells may contribute to the identification of the cell types of origin in prostatic diseases.

Stem/progenitor cells maintain homeostasis in the adult prostate epithelium and regenerate prostate epithelium after castration, as well as initiate prostate tumorigenesis after targeting by carcinogens ${ }^{1,38,49,50}$. Our finding that OLFM4-knockout RWPE1 cells exhibited enhanced proliferation of the CD44+/CD49F+ stem/ progenitor-like cell population, shifts stem/progenitor-like cell division to favor symmetric division and differentiated into higher levels PSA expression cells in organoid assays when compared with OLFM4-wild RWPE1 cells. OLFM4-knockout GFP reporter RWPE1 stem/progenitor-like cells exhibited enhanced self-renewal and disrupted differentiation suggests that OLFM4 plays a role in regulating self-renewal and differentiation of stem/progenitor cells in the normal prostate epithelium and in the initiation of malignant progression. Indeed, OLFM4-expressing prostate stem/progenitor cells were immortalized in the RWPE1 cell line, which mimics the initiation of malignant progression of prostate epithelial cells. Therefore, OLFM4-expressing stem/progenitor cells may be targets of oncogenic transformation in the progression of prostate cancers. 
We explored the molecular mechanisms by which OLFM4 mediates stem/progenitor cell proliferation and differentiation using RNA sequencing, bioinformatics, and whole-genome transcriptome analysis approaches. This analysis revealed that WNT/APC/MYC signaling and MYC target genes were enriched in OLFM4-knockout GFP reporter RWPE1 cells. These findings are similar to previously published gene array data that show Wntsignaling pathway genes were upregulated in colon tissues from Olfm4-knockout $A p c^{\mathrm{Min} /+} \mathrm{mice}^{51}$. Interestingly, we previously found that OLFM4 is a Wnt-signaling target gene and negatively regulates the Wnt-signaling pathway through directly binding to frizzled-7 and frizzled-10 in colon-cancer cells ${ }^{51}$. We conclude that knockout of OLFM4 eliminates a negative control factor of WNT/APC/MYC signaling genes and MYC target genes that regulate multiple biological process and signaling pathways. Therefore, loss of OLFM4 enhances proliferation and disrupts differentiation of prostate stem/progenitor cells (Supplementary Fig. S8). Taken together, these findings suggest that OLFM4 plays an important role in proliferation and differentiation of prostate stem/progenitor cells through majority mediation of WNT/APC/MYC signaling.

\section{Methods}

RWPE1 cell line. The immortalized human normal prostate epithelial cell line RWPE1 was purchased from the American Type Culture Collection (ATCC, CRL-11609) and maintained in culture in prostate epithelial cell basal medium (ATCC PCS-440-030) supplemented with the prostate epithelial cell growth kit (ATCC PCS-440040) in T25 flasks. The RWPE1 cell lines were authenticated and characterized by ATCC, which uses morphology, karyotyping, and PCR-based approaches to confirm the identity of cell lines. All cells were maintained at lower passages.

Single-cell RNA sequencing for RWPE1 cells. Single RWPE1 cells (passage 2) were prepared and sequenced using the Chromium Single Cell 3' Reagent Kit V3 (10 X Genomics) following the Single Cell Protocols Guide from the manufacturer. RNA sequencing was performed by the DNA Sequencing and Genomics Core Facility at the National Heart, Lung, and Blood Institute using an Illumina Hi-Seq instrument.

Single-cell RNA sequencing data analysis. The barcodes, genes, and matrix files resulting from Cell Ranger utility as applied to 10X specific single-cell RNA sequencing for normal adult human prostate with GEO submission number (GSE117403) ${ }^{8}$ were downloaded and analyzed using Seurat R package ${ }^{52}$. The singlecell data-based gene co-regulation network was generated by correlating the dimensionality reduction coordinates. This approach is derived from the Functional Gene mRNA (FGM) profiling method ${ }^{53}$. The FGM profiling method applies principal component analysis (PCA) on the correlation matrix to obtain gene loadings. Finding the significant number of PC's and correlating genes based on these loadings is the central idea behind FGM profiling method to obtain co-regulation networks. Since scRNA-seq data sets yield in quantified transcriptomes that are noisy, diffusion maps were used instead of PCA for dimensionality reduction (www.helmholtz-muenc hen.de/icb/destiny). In our network generation approach, we identify significant diffusion map components $(\mathrm{DMC})$, and correlate the significant DMC's and retain high correlations (absolute Pearson's correlation $\geq 0.65$ ) to obtain the network.

Fluorescent immunohistochemistry and immunocytochemistry. Unstained paraffin section slides of human prostate cancer tissues were purchased from The Cooperative Human Tissue Network (CHTN, Mid-Atlantic Division). Fluorescent immunohistochemistry on paraffin sections or immunocytochemistry on RWPE1 cells was performed as described previously ${ }^{8}$. Images were obtained using the Zeiss 880 Confocal Microscope (inverted). Primary antibodies were used for staining: anti-OLFM4 (OLFM4 (D1E4M) Rabbit mAb, Cell Signaling Technology Inc., \#14369); anti-CK13 (clone EPR3671, Abcam, Cat\# ab92551); anti-CD44 (NBP1-47386, 8E2F3, Novus Biologicals); chicken anti-CK5 (Biolegend, Cat\#905901); anti-SCGB1A1 (clone 394324, Novus Biologicals Cat\# MAB4218-SP); anti-FOXA1 (ab 55178, Lot\# GR3241742-2, Abcam); and mouse anti-CK8 (MMS-162p-250, 1E8, Covance); anti-AR (N-20, SC-816, Santa Cruz Biotechnology); anti-PSA (5G6, SC-52172, Santa Cruz Biotechnology); anti-P63 ( D9L7L, Rabbit mAb, \#39692, Cell Signaling Technology Inc.); anti-CK17/19 (D4G2, Rabbit mAb, \#12434, Cell Signaling Technology Inc); anti-CK14 (\#MA5-11599, invitrogen); anti-SYN (SY38, ab8049-1, abcam); and anti-MYC (D3N8F, Rabbit mAb, \#13987, Cell Signaling Technology Inc).

The images were processed with Fiji 3 software for 2D images and Imais 64-9.21 for 3D images. The combined picture panels were assembled with Adobe Photoshop CC 2017.

Generation of OLFM4-wild or OLFM4-knockout GFP reporter RWPE1 cells. To generate OLFM4wild-GFP reporter cells and OLFM4-knock out-GFP reporter cells, we transfected the CRISPR/Cas 9 OLFM4 activation or knock out plasmids into the RWPE1 OLFM4-wild cells (Supplementary Fig. S4). The Double Nickase Plasmid CRISPR/Cas9 knockout plasmid (sc-403599-NIC and sc-403599-NIC-2), the CRISPR/Cas9 activation plasmid (sc-403599-ACT and sc-403599-ACT-2), and the GC-1 HDR-plasmid (h) (sc-403599-HDR) for the human OLFM4 gene were purchased from Santa Cruz Biotechnology. The plasmid carried gRNA sequencing of the OLFM4 gene as follows: OLFM4 gRNA sequencing: sc-403599-ACT GC-1 CRISPR Activation Plasmid (h): AATGTTTGGCAGGGGATATC (Intron 4; 18747 bp); sc-403599-ACT-2 GC-1 CRISPR Activation Plasmid (h2): CTTTCAAGGAAGTACCAAGT (Intron 2; 6443 bp); sc-403599-NIC GC-1 Double Nickase Plasmid (h): cgtggacagagtggaacgct (Exon 2; 25593 bp); sc-403599-NIC GC-1 Double Nickase Plasmid (h): cagggaaacagagcactggc (Intron 2; 5844 bp); sc-403599-NIC-2 GC-1 Double Nickase Plasmid (h2): tccagccgcagcttaggcag (Exon 1; 9-205 bp); sc-403599-NIC-2 GC-1 Double Nickase Plasmid (h2): gctggagcccgacctggagc (Intron 1; 1051 bp). The plasmids were transfected into RWPE1 cells using Lipofectamine 2000 reagents (Invitrogen) according to 
the manufacturer's instructions. The cells were cultured in the presence of $5 \mu \mathrm{g} / \mathrm{ml}$ puromycin (Invitrogen) to establish stable cell lines.

Cell sorting and FACS analysis. OLFM4-wild GFP reporter and OLFM4-knockout GFP reporter RWPE1 cells were grown in prostate epithelium growth medium (ATCC) to $80 \%$ confluence in T25 flasks. Cells were harvested by trypsinization, removed from flasks, and centrifuged at $1000 \mathrm{rpm}$ for $5 \mathrm{~min}$ at room temperature.

For cell sorting, $1-2 \times 10^{7}$ cells $/ \mathrm{ml}$ were washed once with medium, then resuspended in $1 \mathrm{ml}$ medium. AntiGFP Alexa Fluor 488-conjugated antibody (1:200; Invitrogen) was added to the cell suspension and incubated on ice for $30 \mathrm{~min}$. After washing and filtering with a cell strainer $(100 \mu \mathrm{m}$ filter, BD Falcon), FACS-based cell sorting was performed with BD Aria (BD Biosciences).

For FACS analysis, $1-2 \times 10^{6}$ cells/ml were resuspended in $1 \mathrm{ml}$ PBS and stained with the following antibodies: anti-GFP Alexa Fluor 488-conjugated antibody (1:200; Invitrogen) anti-CD49F PE-conjugated antibody (1:200, eBioscience); anti-CD44 PE-conjugated antibody (1:200, eBioscience); anti-CD26 PE-conjugated antibody (1:100, eBioscience); or anti-CD24 PE-conjugated antibody (1:100, eBioscience) for $1 \mathrm{~h}$ at room temperature, mixing with rotation. The cells were then washed with PBS once and resuspended in $0.5 \mathrm{ml} \mathrm{PBS}$, then analyzed by flow cytometry (BD FACSCalibur, BD Biosciences).

Prostate sphere-formation assay. Prostate sphere-formation assays were performed following a previously described protocol ${ }^{54}$. Briefly, $1 \times 10^{4}$ OLFM4-wild or OLFM4-knockout GFP reporter RWPE1 cells were suspended in $50 \mu \mathrm{l}$ growth medium and mixed with $50 \mu \mathrm{l}$ Matrigel, then cultured in 12-well plates for up to 14 days. For cells treated with $100 \mathrm{nM}$ DHT (Sigma-Aldrich, \#A8380) or 0.1 to $1 \mu \mathrm{M}(+)$-JQ1 (Sigma-Aldrich, \#SML1524), the treatment medium was replaced with fresh medium containing the treatment reagent every 2 days. Dimethyl sulfoxide (DMSO) was used as a vehicle control for all treatment reagents. Images of spheres were captured with an AX10 cam 503 mono or GFP AX10 Cam 105 Color with a ZEISS microscope (AX 10) and ZEISS software for different timepoints, and GFP-positive colonies larger than $50 \mu \mathrm{m}$ in diameter were counted.

Organoid culture. Organoid culture was performed following the protocol published previously by Drost et al. ${ }^{1}$ Briefly, $4 \times 10^{5}$ OLFM4-wild or OLFM4-knockout GFP reporter RWPE1 cells were placed with $40 \mu \mathrm{l}$ Matrigel in the center of each well in a 24-well plate. Human organoid culture medium was prepared following the protocol and $0.5 \mathrm{ml}$ was added to each well. Organoid growth was traced from day 1 to day 12 by taking pictures of GFP-expressing single cells using a ZEISS AXIO microscope with either a GFP filter or using a light field. Organoid images were processed with Adobe Photoshop software. For immunofluorescence staining, organoids that had been cultured for 12 days were fixed with $10 \%$ formalin solution (Sigma-Aldrich, \# HT5011) in PBS at room temperature for $1 \mathrm{~h}$, then changed into $70 \%$ ethanol overnight. After paraffin embedding, $5-\mu \mathrm{m}$ sections of organoids were cut, and paraffin section slides used for fluorescent immunohistochemistry.

Colony-formation assay and (+)-JQ1 treatment. RWPE1 cells were plated at $1 \times 10^{4}$ cells/well in precoated six-well plates and grown overnight in prostate epithelial cell basal medium supplemented with the prostate epithelial cell growth kit. The next day, the medium was changed to treatment medium containing DMSO (vehicle control) or 1-10 $\mu \mathrm{M}(+)-\mathrm{JQ} 1$ and cultured for 7 days. The treatment medium was replaced with fresh medium containing the treatment reagent every 2 days. The cells were then stained with the Diff-Quik Stain Set (Data Behring Inc.) and photographed. The cell images were photographed under light (AX10 cam 503 mono) or fluorescent (AX10 Cam 105 Color) conditions with a ZEISS microscope (AX 10) and ZEISS software for different timepoints. All images were processed with Adobe Photoshop for presentation.

Bulk-cell RNA sequencing and data analysis. Total RNA was purified from GFP-sorted bulk RWPE1 cells using RNeasy plus Mini kits. RNA sequencing was performed by the DNA Sequencing and Genomics Core Facility at the National Heart, Lung, and Blood Institute using an Illumina Hi-Seq instrument. Briefly, sequencing libraries were constructed from 100-500 ng of total RNA using the Illumina TruSeq Stranded Total RNA kit with Ribo-Zero following the manufacturer's instructions. The fragment size of the RNAseq libraries was verified using an Agilent 2100 Bioanalyzer and the concentrations determined using a Qubit instrument (LifeTech). The libraries were loaded onto the Illumina HiSeq 3000 for $2 \times 75$ bp paired-end read sequencing. The FASTQ files were generated using Illumina bcl2fastq conversion software (https://support.illumina.com/sequencing/ sequencing_software/bcl2fastq-conversion-software.html) for further whole-genome transcriptome analysis.

Quality control of FASTQ files was assessed using the FastQC toolkit (v0.11.5) using default parameters. The paired-end reads were aligned against the human reference genome (GENCODE GRCh38) using HISAT2 (v2.0.5) alignment ${ }^{55}$. Gene-level read counts were produced by featureCounts (v1.5.2) $)^{56}$ using only uniquely mapped, paired-end, reversely stranded reads. Differential-expression analysis at the gene level was conducted using limma-voom open-source R packages ${ }^{57,58}$. TMM (Trimmed Mean of M values) normalization was conducted, and normalized factors were estimated for each sample ${ }^{59}$. The $1 \mathrm{mFit}$ package in limma-voom was used to fit linear models for each gene to calculate log2-fold changes and $p$ values using the normalized factors as weights in the model. To account for multiple testing, the false discovery rate (FDR) via the Benjamani-Hochberg algorithm ${ }^{60}$ was calculated ${ }^{61}$.

We characterized differentially expressed genes (DEGs) with respect to both gene ontology (GO) and pathway enrichment to assess the functional association of the DEGs. Gene set enrichment for GO $^{62}$ including biological process, cellular component, and molecular function analysis, Kyoto Encyclopedia of Genes and Genomes (KEGG) analysis ${ }^{63}$, Panther Database ${ }^{64}$, and Wikipathway ${ }^{65}$ were performed using WebGestalt ${ }^{66}$ using $p<0.05$ as 
the cut-off criterion. Gene set enrichment analysis (GSEA) was conducted to identify the significantly upregulated and downregulated pathways between high and low DEGs using FDR $<0.05$ as the cut-off criterion.

Statistical analysis. Student's t-test was used to analyze differences between groups. $p$ values $<0.05$ were considered statistically significant.

\section{Data availability}

RNA sequencing data that support the findings of this study have been deposited in the GEO database, with the accession code GSE126162 (https://www.ncbi.nlm.nih.gov/geo/query/acc.cgi?acc=GSE126162).

Received: 6 August 2020; Accepted: 12 November 2020

Published online: 14 December 2020

\section{References}

1. Isaacs, J. T. \& Coffey, D. S. Etiology and disease process of benign prostatic hyperplasia. Prostate Suppl 2, 33-50 (1989).

2. DeKlerk, D. P. \& Coffey, D. S. Quantitative determination of prostatic epithelial and stromal hyperplasia by a new technique. Biomorphometrics. Invest. Urol. 16, 240-245 (1978).

3. Taylor, R. A., Toivanen, R. \& Risbridger, G. P. Stem cells in prostate cancer: treating the root of the problem. Endocr. Relat. Cancer 17, R273-285. https://doi.org/10.1677/erc-10-0145 (2010).

4. Collins, A. T., Berry, P. A., Hyde, C., Stower, M. J. \& Maitland, N. J. Prospective identification of tumorigenic prostate cancer stem cells. Can. Res. 65, 10946-10951. https://doi.org/10.1158/0008-5472.Can-05-2018 (2005).

5. Richardson, G. D. et al. CD133, a novel marker for human prostatic epithelial stem cells. J. Cell Sci. 117, 3539-3545. https://doi. org/10.1242/jcs.01222 (2004).

6. Goldstein, A. S. et al. Trop2 identifies a subpopulation of murine and human prostate basal cells with stem cell characteristics. Proc. Natl. Acad. Sci. USA 105, 20882-20887. https://doi.org/10.1073/pnas.0811411106 (2008).

7. $\mathrm{Hu}, \mathrm{W}$. Y. et al. Isolation and functional interrogation of adult human prostate epithelial stem cells at single cell resolution. Stem Cell Res. 23, 1-12. https://doi.org/10.1016/j.scr.2017.06.009 (2017).

8. Henry, G. H. et al. A cellular anatomy of the normal adult human prostate and prostatic urethra. Cell Rep. 25, 3530-3542.e3535. https://doi.org/10.1016/j.celrep.2018.11.086 (2018).

9. McCray, T., Moline, D., Baumann, B., Vander Griend, D. J. \& Nonn, L. Single-cell RNA-Seq analysis identifies a putative epithelial stem cell population in human primary prostate cells in monolayer and organoid culture conditions. Am. J. Clin. Exp. Urol. 7, 123-138 (2019).

10. Wang, Y., Hayward, S., Cao, M., Thayer, K. \& Cunha, G. Cell differentiation lineage in the prostate. Differ. Res. Biol. Divers. 68, 270-279 (2001).

11. De Marzo, A. M., Meeker, A. K., Epstein, J. I. \& Coffey, D. S. Prostate stem cell compartments: expression of the cell cycle inhibitor p27Kip1 in normal, hyperplastic, and neoplastic cells. Am. J. Pathol. 153, 911-919. https://doi.org/10.1016/s0002-9440(10)65632 -5 (1998).

12. Uzgare, A. R., Xu, Y. \& Isaacs, J. T. In vitro culturing and characteristics of transit amplifying epithelial cells from human prostate tissue. J. Cell. Biochem. 91, 196-205. https://doi.org/10.1002/jcb.10764 (2004).

13. Signoretti, S. et al. p63 is a prostate basal cell marker and is required for prostate development. Am. J. Pathol. 157, 1769-1775. https ://doi.org/10.1016/s0002-9440(10)64814-6 (2000).

14. Leong, K. G., Wang, B. E., Johnson, L. \& Gao, W. Q. Generation of a prostate from a single adult stem cell. Nature 456, 804-808. https://doi.org/10.1038/nature07427 (2008).

15. Lawson, D. A., Xin, L., Lukacs, R. U., Cheng, D. \& Witte, O. N. Isolation and functional characterization of murine prostate stem cells. Proc. Natl. Acad. Sci. USA 104, 181-186. https://doi.org/10.1073/pnas.0609684104 (2007).

16. Burger, P. E. et al. Sca-1 expression identifies stem cells in the proximal region of prostatic ducts with high capacity to reconstitute prostatic tissue. Proc. Natl. Acad. Sci. USA 102, 7180-7185. https://doi.org/10.1073/pnas.0502761102 (2005).

17. Karthaus, W. R. et al. Identification of multipotent luminal progenitor cells in human prostate organoid cultures. Cell 159, 163-175. https://doi.org/10.1016/j.cell.2014.08.017 (2014).

18. Chua, C. W. et al. Single luminal epithelial progenitors can generate prostate organoids in culture. Nat. Cell Biol. 16(951-961), 951-954. https://doi.org/10.1038/ncb3047 (2014).

19. Li, H. et al. Telomerase-immortalized non-malignant human prostate epithelial cells retain the properties of multipotent stem cells. Exp. Cell Res. 314, 92-102. https://doi.org/10.1016/j.yexcr.2007.08.011 (2008).

20. Miki, J. et al. Identification of putative stem cell markers, CD133 and CXCR4, in hTERT-immortalized primary nonmalignant and malignant tumor-derived human prostate epithelial cell lines and in prostate cancer specimens. Can. Res. 67, 3153-3161. https:// doi.org/10.1158/0008-5472.Can-06-4429 (2007).

21. Rhim, J. S. et al. Stepwise immortalization and transformation of adult human prostate epithelial cells by a combination of HPV-18 and v-Ki-ras. Proc. Natl. Acad. Sci. USA 91, 11874-11878. https://doi.org/10.1073/pnas.91.25.11874 (1994).

22. Kharaishvili, G. et al. Wnt signaling in prostate development and carcinogenesis. Biomed. Pap. Med. Fac. Univ. Palacky Olomouc Czechoslov. 155, 11-18. https://doi.org/10.5507/bp.2011.016 (2011).

23. He, T. C. et al. Identification of c-MYC as a target of the APC pathway. Science (New York, N.Y.) 281, 1509-1512. https://doi. org/10.1126/science.281.5382.1509 (1998).

24. Dang, C. V. et al. The c-Myc target gene network. Semin. Cancer Biol. 16, 253-264. https://doi.org/10.1016/j.semcancer.2006.07.014 (2006).

25. Bretones, G., Delgado, M. D. \& Leon, J. Myc and cell cycle control. Biochim. Biophys. Acta 1849, 506-516. https://doi.org/10.1016/j. bbagrm.2014.03.013 (2015).

26. Nupponen, N. N., Kakkola, L., Koivisto, P. \& Visakorpi, T. Genetic alterations in hormone-refractory recurrent prostate carcinomas. Am. J. Pathol. 153, 141-148. https://doi.org/10.1016/s0002-9440(10)65554-x (1998).

27. Fleming, W. H. et al. Expression of the c-myc protooncogene in human prostatic carcinoma and benign prostatic hyperplasia. Can. Res. 46, 1535-1538 (1986).

28. Buttyan, R., Sawczuk, I. S., Benson, M. C., Siegal, J. D. \& Olsson, C. A. Enhanced expression of the c-myc protooncogene in highgrade human prostate cancers. Prostate 11, 327-337 (1987).

29. Gurel, B. et al. Nuclear MYC protein overexpression is an early alteration in human prostate carcinogenesis. Mod. Pathol. 21, 1156-1167. https://doi.org/10.1038/modpathol.2008.111 (2008).

30. Ellwood-Yen, K. et al. Myc-driven murine prostate cancer shares molecular features with human prostate tumors. Cancer Cell 4, 223-238 (2003).

31. Hubbard, G. K. et al. Combined MYC activation and Pten loss are sufficient to create genomic instability and lethal metastatic prostate cancer. Can. Res. 76, 283-292. https://doi.org/10.1158/0008-5472.Can-14-3280 (2016). 
32. Zhang, J. et al. Identification and characterization of a novel member of olfactomedin-related protein family, hGC-1, expressed during myeloid lineage development. Gene 283, 83-93 (2002).

33. Liu, W. \& Rodgers, G. P. Olfactomedin 4 expression and functions in innate immunity, inflammation, and cancer. Cancer Metastasis Rev. 35, 201-212. https://doi.org/10.1007/s10555-016-9624-2 (2016).

34. Li, H. et al. Deletion of the olfactomedin 4 gene is associated with progression of human prostate cancer. Am. J. Pathol. 183, 1329-1338. https://doi.org/10.1016/j.ajpath.2013.06.028 (2013).

35. Li, H. et al. Olfactomedin 4 downregulation is associated with tumor initiation, growth and progression in human prostate cancer. Int. J. Cancer https://doi.org/10.1002/ijc.32535 (2019).

36. Chen, L. et al. Olfactomedin 4 suppresses prostate cancer cell growth and metastasis via negative interaction with cathepsin $\mathrm{D}$ and SDF-1. Carcinogenesis 32, 986-994. https://doi.org/10.1093/carcin/bgr065 (2011).

37. Montoro, D. T. et al. A revised airway epithelial hierarchy includes CFTR-expressing ionocytes. Nature 560, 319-324. https://doi. org/10.1038/s41586-018-0393-7 (2018).

38. Li, J. J. \& Shen, M. M. Prostate stem cells and cancer stem cells. Cold Spring Harb Perspect. Med. https://doi.org/10.1101/cshpe rspect.a030395 (2018).

39. Drost, J. et al. Organoid culture systems for prostate epithelial and cancer tissue. Nat. Protoc. 11, 347-358. https://doi.org/10.1038/ nprot.2016.006 (2016).

40. Sansom, O. J. et al. Myc deletion rescues Apc deficiency in the small intestine. Nature 446, 676-679. https://doi.org/10.1038/natur e05674 (2007)

41. Cunha, G. R. et al. Development of the human prostate. Differ. Res. Biol. Divers. 103, 24-45. https://doi.org/10.1016/j. diff.2018.08.005 (2018).

42. Toivanen, R. \& Shen, M. M. Prostate organogenesis: tissue induction, hormonal regulation and cell type specification. Dev. Camb. Engl. 144, 1382-1398. https://doi.org/10.1242/dev.148270 (2017).

43. Barros-Silva, J. D. et al. Single-cell analysis identifies LY6D as a marker linking castration-resistant prostate luminal cells to prostate progenitors and cancer. Cell Rep. 25, 3504-3518.e3506. https://doi.org/10.1016/j.celrep.2018.11.069 (2018).

44. Moad, M. et al. Multipotent basal stem cells, maintained in localized proximal niches, support directed long-ranging epithelial flows in human prostates. Cell Rep. 20, 1609-1622. https://doi.org/10.1016/j.celrep.2017.07.061 (2017)

45. Petkova, N. et al. Surface CD24 distinguishes between low differentiated and transit-amplifying cells in the basal layer of human prostate. Prostate 73, 1576-1590. https://doi.org/10.1002/pros.22708 (2013).

46. Zhang, D., Zhao, S., Li, X., Kirk, J. S. \& Tang, D. G. Prostate luminal progenitor cells in development and cancer. Trends Cancer 4, 769-783. https://doi.org/10.1016/j.trecan.2018.09.003 (2018).

47. Wang, X. et al. A luminal epithelial stem cell that is a cell of origin for prostate cancer. Nature 461, 495-500. https://doi.org/10.1038/ nature08361 (2009).

48. Zhang, D. et al. Developing a novel two-dimensional culture system to enrich human prostate luminal progenitors that can function as a cell of origin for prostate cancer. Stem Cells Transl. Med. 6, 748-760. https://doi.org/10.5966/sctm.2016-0243 (2017).

49. Shen, M. M. \& Abate-Shen, C. Molecular genetics of prostate cancer: new prospects for old challenges. Genes Dev. 24, 1967-2000. https://doi.org/10.1101/gad.1965810 (2010).

50. Choi, N., Zhang, B., Zhang, L., Ittmann, M. \& Xin, L. Adult murine prostate basal and luminal cells are self-sustained lineages that can both serve as targets for prostate cancer initiation. Cancer Cell 21, 253-265. https://doi.org/10.1016/j.ccr.2012.01.005 (2012).

51. Liu, W. et al. Olfactomedin 4 deletion induces colon adenocarcinoma in $\mathrm{Apc}(\mathrm{Min} /+)$ mice. Oncogene 35, 5237-5247. https://doi. org/10.1038/onc.2016.58 (2016).

52. Stuart, T. et al. Comprehensive integration of single-cell data. Cell 177, 1888-1902.e1821. https://doi.org/10.1016/j.cell.2019.05.031 (2019).

53. Jiang, P. \& Liu, X. S. Big data mining yields novel insights on cancer. Nat. Genet. 47, 103-104. https://doi.org/10.1038/ng.3205 (2015).

54. Lukacs, R. U., Goldstein, A. S., Lawson, D. A., Cheng, D. \& Witte, O. N. Isolation, cultivation and characterization of adult murine prostate stem cells. Nat. Protoc. 5, 702-713. https://doi.org/10.1038/nprot.2010.11 (2010).

55. Kim, D., Langmead, B. \& Salzberg, S. L. HISAT: a fast spliced aligner with low memory requirements. Nat. Methods 12, 357-360. https://doi.org/10.1038/nmeth.3317 (2015).

56. Mertz, J. A. et al. Targeting MYC dependence in cancer by inhibiting BET bromodomains. Proc. Natl. Acad. Sci. USA 108, 1666916674. https://doi.org/10.1073/pnas.1108190108 (2011).

57. Liao, Y., Smyth, G. K. \& Shi, W. The Subread aligner: fast, accurate and scalable read mapping by seed-and-vote. Nucleic Acids Res. 41, e108. https://doi.org/10.1093/nar/gkt214 (2013).

58. Law, C. W., Chen, Y., Shi, W. \& Smyth, G. K. voom: Precision weights unlock linear model analysis tools for RNA-seq read counts. Genome Biol. 15, R29. https://doi.org/10.1186/gb-2014-15-2-r29 (2014).

59. Robinson, M. D. \& Oshlack, A. A scaling normalization method for differential expression analysis of RNA-seq data. Genome Biol. 11, R25. https://doi.org/10.1186/gb-2010-11-3-r25 (2010).

60. Abate-Shen, C. \& Shen, M. M. Molecular genetics of prostate cancer. Genes Dev. 14, 2410-2434 (2000).

61. Madar, V. \& Batista, S. FastLSU: a more practical approach for the Benjamini-Hochberg FDR controlling procedure for huge-scale testing problems. Bioinformatics 32, 1716-1723. https://doi.org/10.1093/bioinformatics/btw029 (2016).

62. Ashburner, M. et al. Gene ontology: tool for the unification of biology. The gene ontology consortium. Nat. Genet. 25, 25-29. https ://doi.org/10.1038/75556 (2000).

63. Ogata, H. et al. KEGG: kyoto encyclopedia of genes and genomes. Nucleic Acids Res. 27, 29-34. https://doi.org/10.1093/nar/27.1.29 (1999).

64. Mi, H. et al. The PANTHER database of protein families, subfamilies, functions and pathways. Nucleic Acids Res. 33, D284-288. https://doi.org/10.1093/nar/gki078 (2005).

65. Slenter, D. N. et al. WikiPathways: a multifaceted pathway database bridging metabolomics to other omics research. Nucleic Acids Res. 46, D661-d667. https://doi.org/10.1093/nar/gkx1064 (2018).

66. Liao, Y., Wang, J., Jaehnig, E. J., Shi, Z. \& Zhang, B. WebGestalt 2019: gene set analysis toolkit with revamped UIs and APIs. Nucleic Acids Res. 47, W199-w205. https://doi.org/10.1093/nar/gkz401 (2019).

\section{Acknowledgements}

This work was supported by the Intramural Research Program, National Institutes of Health/National Institute of Diabetes and Digestive and Kidney Diseases. We thank Drs. Yuesheng Li, Poching Liu, and Yan Luo (DNA Sequencing and Genomics Core Facility, National Heart, Lung, and Blood Institute) for help with RNA sequencing. We thank Dr. J. Philip McCoy (Flow Cytometry Core Facility, National Heart, Lung, and Blood Institute) for help with GFP-based cell sorting. We thank Drs. Christian A. Combs and Daniela Malide (Light Microscopy Core Facility, National Heart, Lung, and Blood Institute, National Institutes of Health) for help with image processes. 


\section{Author contributions}

Conception and design: H.L. and G.P.R. Development of methodology: H.L., W.L., J.Z., K.C. Acquisition of data (provided animals, acquired and managed patients, provided facilities, etc.): H.L. and G.P.R. Analysis and interpretation of data (e.g., statistical analysis, biostatistics, computational analysis): H.L., V.C., M.P., G.P.R. Writing, review, and/or revision of the manuscript: H.L. and G.P.R. Administrative, technical, or material support (i.e., reporting or organizing data, constructing databases): H.L., V.C., M.P., G.P.R. Study supervision: G.P.R.

\section{Competing interests}

The authors declare no competing interests.

\section{Additional information}

Supplementary Information The online version contains supplementary material available at https://doi. org/10.1038/s41598-020-78774-5.

Correspondence and requests for materials should be addressed to G.P.R.

Reprints and permissions information is available at www.nature.com/reprints.

Publisher's note Springer Nature remains neutral with regard to jurisdictional claims in published maps and institutional affiliations.

(c) Open Access This article is licensed under a Creative Commons Attribution 4.0 International License, which permits use, sharing, adaptation, distribution and reproduction in any medium or format, as long as you give appropriate credit to the original author(s) and the source, provide a link to the Creative Commons licence, and indicate if changes were made. The images or other third party material in this article are included in the article's Creative Commons licence, unless indicated otherwise in a credit line to the material. If material is not included in the article's Creative Commons licence and your intended use is not permitted by statutory regulation or exceeds the permitted use, you will need to obtain permission directly from the copyright holder. To view a copy of this licence, visit http://creativecommons.org/licenses/by/4.0/.

This is a U.S. Government work and not under copyright protection in the US; foreign copyright protection may apply 2020 